\title{
Prediction of missing links based on community relevance and ruler inference
}

Jingyi Ding*, Licheng Jiao, Jianshe Wu, Fang Liu

Key Laboratory of Intelligent Perception and Image Understanding of Ministry of Education, International Research Center for Intelligent Perception and Computation,

Xidian University, Xi'an, Shaanxi Province 710071, China

(* Corresponding author, Tel: +86 15929737040.E-mail addresses: jyding87@163.com)

\begin{abstract}
The link prediction algorithm which based on node similarity is the research hotspot in recent years. In addition, there are some methods which based on the network community structure information to predict the missing links, however, these studies only concerned about the obvious information between different communities such as direct links. We found that it is hard to predict the missing links if the two communities have little direct connections. In fact, there is similarity between communities such as the similarity between nodes and this similarity is significant for prediction. So, we define a community similarity feature which named community relevance by using not only the obvious information but also the latent information between different communities in this paper. Then a novel algorithm which based on the community relevance and ruler inference is proposed to predict missing links. In this method, we extract the community structure by using the local information of the network first. Next, calculate the relevance of each pair of communities by using the new community relevance indices. Finally, a simple prediction model which based on ruler inference is applied to estimate the probability of the missing links. It is shown that the proposed method has more effective prediction accuracy and the community relevance features improve the predictor with low time complexity, with experiments on benchmark networks and real-world networks in different scales, and compared with other ten sate of the art approaches.

Keywords: link prediction, community detection, community relevance, ruler inference, complex networks
\end{abstract}

\section{Introduction}

A network is composed of different individuals and their static or dynamic relations. These relations can be friendship among people or physical interaction among genes. Link prediction, predicting missing links or future structure of the network from the observed structure, is an important task in link mining [1, 2], it is also an effective method and means for data mining. Link prediction 
helps us not only to understand the evolution mechanism of the complex networks theoretically [3, 4, 5], but also to solve very important issues in applications. For example, it can be used in the field of information integration, social network analysis, recommender systems [6] and bioinformatics.

We can divide the existing methods for link prediction into three categories. The first kind of methods define a measure of similarity between two nodes in the network, considering that links between two nodes with more similar are of higher existing probability [7,8]. The second kind of approaches based on the maximum likelihood estimation [9]. The third kind of algorithms based on machine learning techniques $[10,11]$. There are also many studies related to link prediction focusing on more complicated networks, like directed and weighted networks [12, 13].

Link prediction in networks is one of the most important issues in complex networks. Previous proposed algorithms are mainly based on Markovian chains and machine learning. Then, a prediction method based on node similarity was proposed in 1998 [7]. Owing to the low complexity and high prediction accuracy, the link prediction algorithm which based on node similarity is the research hotspot in recent years. After that Nowell and Kleinberg summarize many similarity measures in ref. [8]. One kind of measures based on node neighborhoods, which concern the local structure of the networks. Another based on all paths of a network, which consider the global structure of the networks. However, the first kind of measures may not be enough for edge prediction, and the second measures have a high computational complexity. Afterwards, it's found that the hierarchical organization information [14, 15] may indeed provide insights for link prediction. So, a maximum likelihood method which considered the hierarchical organization information of the network was proposed by Clauset, Moore and Newman in 2008 [9]. This algorithm combined with Monte-Carlo algorithm and fit a hierarchical model on a network graph to make link predictions. The results showed that the performance is well if the network has obvious hierarchical organization. However, the disadvantage of the algorithm is the runtime required increase exponentially as the number of vertices increases in the worst case. Next, another representative method which named stochastic block model was proposed by Guimerà in 2009 [16]. In the stochastic block model, nodes are clustered into groups and the probability that two nodes are connected depends only on the groups to which they belong. The advantages of this method are that we can identify not only missing interactions but also spurious interactions. Besides, we can also generate a reconstructed network from a single observed network by using this model. Unfortunately, the algorithm is very time consuming because the number of different 
partitions of $N$ elements grows faster than any finite power of $N$. The time complexity of the method increase exponentially as the number of vertices increases in the worst case. Later, a method based on the community structure was proposed by Yan in 2012 [17]. It's proved that the community structure information is of great significance for link prediction. In the paper, the missing links are divided into two different parts, links in the same community nodes and links between different communities nodes, they tried to rank them by using the same node similarity indices, considering that links in the same community nodes are more similar and have higher existing probability. From this method, we can find that the existing probability of the missing links is zero when the similarity of two terminal nodes which in different communities is zero. This is different from the real phenomenon. Afterwards, Cannistraci et al. proposed a new method which based on the strategy of link-community in 2013 [4]. His algorithm introduces a new philosophy in the formulation of neighborhood-based indices which is named CAR-based variant. CAR suggests that two nodes are more likely to link together if their common-first-neighbors are members of a strongly inner-linked cohort. The time complexity of the method is $O\left(N^{2}\right) \sim O\left(N^{4}\right)$. Next, an algorithm based on the multi-resolution community structure information of the network was proposed by Ding et. al. in 2014 [18]. Instead of the accurate classification result obtained by a general community detection algorithm, the proposed method just needs the results obtained under different resolutions. The time complexity of the method is $O\left(k N^{2}\right)$. However, the performance of the algorithm is not good enough when the networks have too many hub nodes or the clustering coefficient is too large. In 2015, Lü et. al. proposed a structural perturbation method for link prediction that is more accurate and robust [19]. Their fundamental assumption is that missing links are difficult to predict if their addition causes huge structural changes, and thus network is highly predictable if the removal or addition of a set of randomly selected links does not significantly change the network's structural features. The advantage of this method is that its predictions are consistently more robust. The disadvantage is high time complexity. The time complexity is $O\left(N^{3}\right)$.

Then, we summarize the advantages, the disadvantages and the time complexity of the algorithms and show them in Table 1.

\section{Table 1}

The advantages, the disadvantages and the time complexity of the algorithms

\begin{tabular}{|c|l|l|c|}
\hline algorithm & \multicolumn{1}{|c|}{ advantages } & \multicolumn{1}{c|}{ disadvantages } & Time complexity \\
\hline CN/JC & (1)Low complexity & (1)Treat each common & $O\left(N^{2}\right)$ \\
\hline
\end{tabular}




\begin{tabular}{|c|c|c|c|}
\hline & & $\begin{array}{l}\text { neighbor equally } \\
\text { (2)General accuracy }\end{array}$ & \\
\hline AA/RA & $\begin{array}{l}\text { (1)Differentiate the } \\
\text { contributions of different } \\
\text { neighbors } \\
\text { (2)Low complexity }\end{array}$ & (1)General accuracy & $O\left(N^{2}\right)$ \\
\hline $\begin{array}{c}\text { CAR_CN/JC/AA } \\
/ \text { RA }\end{array}$ & $\begin{array}{l}\text { (1)Consistent robustness } \\
\text { (2)Proposed a } \\
\text { local-community paradigm }\end{array}$ & $\begin{array}{l}\text { (1)Network structure } \\
\text { constraint }\end{array}$ & $O\left(N^{2}\right) \sim O\left(N^{4}\right)$ \\
\hline Yan's algorithm & $\begin{array}{l}\text { (1)Using the community } \\
\text { structure information }\end{array}$ & $\begin{array}{l}\text { (1)Network structure } \\
\text { constraint. } \\
\text { (2)The complexity of } \\
\text { community division } \\
\text { method has great influence } \\
\text { on the whole algorithm. }\end{array}$ & $\begin{array}{l}\text { It takes } O\left(N^{2}\right) \\
\text { to calculate the } \\
\text { probability of } \\
\text { missing links. }\end{array}$ \\
\hline HSM & $\begin{array}{l}\text { (1) Uncovers the hidden } \\
\text { hierarchical organization }\end{array}$ & $\begin{array}{l}\text { (1)Network structure } \\
\text { constraint } \\
\text { (2)High time complexity }\end{array}$ & $\begin{array}{l}\text { In the worst case, } \\
\text { it takes } \\
\text { exponential time } \\
\text { to sample } \\
\text { dendrograms }\end{array}$ \\
\hline SBM & $\begin{array}{l}\text { (1)Identify possible spurious } \\
\text { links, } \\
\text { (2) Network reconstruction; } \\
\text { (3) Robust } \\
\text { (4) Flexible }\end{array}$ & (1)High time complexity & $\begin{array}{l}\text { In the worst case, } \\
\text { it takes } \\
\text { exponential time } \\
\text { to sample different } \\
\text { partitions. }^{a^{*}}\end{array}$ \\
\hline SPM & $\begin{array}{l}\text { (1) Robust. } \\
\text { (2) Consistent } \\
\text { (3) Proposed a universal } \\
\text { structural consistency index }\end{array}$ & (1)High time complexity & $O\left(N^{3}\right)^{\mathrm{b}^{*}}$ \\
\hline $\mathrm{CP}$ & $\begin{array}{l}\text { (1)Using the multi- resolution } \\
\text { information of the network } \\
\text { (2) Uncovers the hidden } \\
\text { community structure }\end{array}$ & $\begin{array}{l}\text { (1)Network structure } \\
\text { constraint } \\
\text { (2) The hub nodes and the } \\
\text { clustering coefficient } \\
\text { constraints }\end{array}$ & $O\left(k N^{2}\right)$ \\
\hline $\begin{array}{c}\text { CRCN/CRJC/CR } \\
\text { AA/CRRA }\end{array}$ & $\begin{array}{l}\text { (1)Define the community } \\
\text { relevance feature } \\
\text { (2) Uncovers the hidden } \\
\text { community structure } \\
\text { (3)Effective } \\
\text { (4)Efficient }\end{array}$ & $\begin{array}{l}\text { (1)Network structure } \\
\text { constraint } \\
\text { (2)Degree distributions } \\
\text { constraint }\end{array}$ & $O\left(N^{2}\right)$ \\
\hline
\end{tabular}

a*The number of distinct partitions of $\mathrm{N}$ elements into groups is $\sum_{k=1}^{N} \frac{1}{k !} \sum_{l=1}^{k}\left(\begin{array}{c}k \\ l\end{array}\right)(-1)^{k-l} l^{N}$, which grows faster than any finite power of $\mathrm{N}[16]$. 
$\mathrm{b}^{*}$ The time complexity of computing the eigenvalues of the matrix is $O\left(N^{3}\right)$.

However, above methods did not take into account the relationship between communities. So the main novelty of our work is to study the relationship between different communities and research the effect of this relationship on link prediction. We propose a link prediction method based on the community relevance which is one of the most important community structure features in this paper. Our focus is on the new philosophy in the formulation of community-based indices. We advocate a shift in perspective from nodes to communities, and in particular from nodes similarity to community relevance. Our method works very fast when the data is typical and sparse.

The paper attempts to solve link prediction problem in a new perspective, believing that the information of community relevance can be helpful to improve the predictor. In the former prediction methods, the existing probability of the missing links is zero when the similarity of two terminal nodes which in different communities is zero. However, in real networks, even the similarity of two terminal nodes which in different communities is zero, the existing probability of the missing links is not zero when the two communities have great relevance. For example, in the cooperation network, even members from different teams which the research directions are very similar without any cooperation, the cooperation is still possible to happen in the future. The probability of the cooperation is depending on the closeness of the research directions of their groups. So in this paper, we model this closeness of different groups as the community relevance and focus on how to define the relevance index between communities to make the results more accurate.

The contributions of this work are:

1). Proposed a new mechanism of link prediction which is based on the community relevance. The formulation of the community relevance index defined in this paper is not a single. It's a new philosophy in the formulation of community-based indices.

2). Reveal the community structure of the network.

3) The two assumptions which we use in the prediction model cause the framework can be used to predict the missing links with new nodes.

4) The results indicate that under the same time complexity, our algorithm with higher prediction accuracy. Moreover, it is more suitable for the community networks which degree distributions consistency.

The rest of the paper is organized as follows: Section 2 describes the problem formulation which 
including the description of link prediction, the definition of the community relevance. Section 3.1 introduces the approach that extracting the community structure of the networks. Section 3.2 explains the new community relevance indices which are based on node similarity indices. Section 3.3 describes the way to predicting missing links by using the ruler inference. The experiments and analyses are provided in Section 4. Finally, Section 5 concludes the paper with some discussions and promising future work.

\section{Problem formulation}

\subsection{Description of link prediction}

The link prediction problem is the task to predict the probability of a link exists between a pair of nodes. Given an undirected network $G(V, E)$, where $V$ is the set of vertices and $E$ is the set of edges. Multiple links and self-connections are not allowed. In the link prediction algorithms, we need to predict the probability of nonexistent link between node $x$ and node $y, x, y \in V$. The probability is expressed as score $S_{x y}$. All the nonexistent links are sorted in decreasing order according to their scores, and the links at the top are most likely to exist.

Generally speaking, we do not know which link is the missing or future links, otherwise we do not need to do prediction. Therefore, in order to test the algorithm's accuracy, we randomly divided the observed links $E$ into two parts: the training set $E^{T}$ and the probe set $E^{P}$. Then we attempt to predict missing links based on the training set $E^{T}$ which is treated as known information. Testing the accuracy of our algorithm based on the probe set $E^{P}$. Clearly, the observed links $E=E^{T} \cup E^{P}$ and $E^{T} \cap E^{P}=$ $\emptyset$.

\subsection{Community relevance}

Generally speaking, an undirected network can be described as $G(V, E)$, where $V=\left\{v_{1}, v_{2}, \ldots, v_{n}\right\}$ represents the set of vertices in the graph and $E=\left\{\left(v_{i}, v_{j}\right) \mid v_{i} \in V, v_{j} \in V\right\}$ represents the set of edges. The community set can be defined as $C=\left\{c_{1}, c_{2}, \ldots, c_{m}\right\}$. The symbol $\Gamma\left(v_{i}\right)$ indicates the neighbors of the vertex $v_{i}$ and $\Gamma\left(c_{i}\right)$ indicates the neighbors of the community $\quad c_{i} \quad . \quad$ We $\quad$ use $\quad V\left(c_{i}\right)=\left\{v \mid v \in V, c_{i} \in C(v)\right\} \quad$ and $E\left(c_{i}\right)=\left\{(v, w) \mid(v, w) \in E, c_{i} \in C((v, w))\right\}$ to represent the sum of the nodes in community $c_{i}$ and the sum of the edges inside community $c_{i}$. The symbol $L\left(c_{i}, \overline{c_{l}}\right)$ expresses the sum of the edges between the community $c_{i}$ and $\bar{c}_{l}$. Mathematically, a network can be represented by an adjacency matrix $A=$ $\left(A_{i j}\right)_{n \times n}$, details a connection between nodes $i$ and $j$, which will normally be 0 or $1, d_{i}=\sum_{j} A_{i j}$ is 
the degree of the node $i$. If $i \subset c_{i}$, we can split the total degrees of $i$ as $d_{i}=d_{i}\left(c_{i}\right)=d_{i}^{i n}\left(c_{i}\right)+$ $d_{i}^{\text {out }}\left(c_{i}\right)$, where $d_{i}^{\text {in }}\left(c_{i}\right)=\sum_{j \in c_{i}} A_{i j}$ is the number of links between $i$ and other nodes belonging to $c_{i}, d_{i}^{\text {out }}\left(c_{i}\right)=\sum_{j \notin c_{i}} A_{i j}$ is the number of connections from $i$ to the rest of the network. So we can found $E\left(c_{i}\right)=\sum_{i \in c_{i}} d_{i}^{i n}\left(c_{i}\right)$ and $L\left(c_{i}, \overline{c_{l}}\right)=\sum_{i \in c_{i}} d_{i}^{\text {out }}\left(c_{i}\right)$. Each community in a complex network can be viewed as a set of vertices. Community detection is the task to find all the sets of vertices. The vertices belonging to the same community with dense connections, while the nodes belonging to different communities with sparse connections [15, 20]. In the past decade, many algorithms have been proposed to detect community structure in complex networks [21-27]. Many algorithms are based on modularity $(Q)$ optimization [28-30]. But, the methods based on $Q$ which calculated by the global information of the network has the resolution limit problem [31-33] and very time consuming. In this paper, we improve the local community divide method which based on node strength [34] to extract the community structure for our link predication.

When we get the community structure of the network from above improvement method, the matrix $C R\left(c_{i}, c_{j}\right),(i, j=1,2, \ldots, m)$ is constructed to compute the community relevance between two communities. The community relevance is an index which used to describe the relationship between two communities. We can see that the matrix of the community relevance is a symmetric matrix.

In this paper we designed four community relevance indices. They are CRCN, CRJC, CRAA and CRRA. Concretes are defined as follows:

1) $\mathrm{CRCN}$ follows the basic ideal that two communities more relevant if they have more common nodes which have connections with them.

$$
C R\left(c_{i}, c_{j}\right)^{C R C N}=\left|\left(\Gamma\left(c_{i}\right) \cup V\left(c_{i}\right)\right) \cap\left(\Gamma\left(c_{j}\right) \cup V\left(c_{j}\right)\right)\right|
$$

2) CRJC measures the probability that both $c_{i}$ and $c_{j}$ share a common neighbors.

$$
C R\left(c_{i}, c_{j}\right)^{C R J C}=\frac{\left|\left(\Gamma\left(c_{i}\right) \cup V\left(c_{i}\right)\right) \cap\left(\Gamma\left(c_{j}\right) \cup V\left(c_{j}\right)\right)\right|}{\left|\left(\Gamma\left(c_{i}\right) \cup V\left(c_{i}\right)\right) \cup\left(\Gamma\left(c_{j}\right) \cup V\left(c_{j}\right)\right)\right|}
$$

3) CRAA and CRRA are two similar indices that predict more accurate when the number of common neighbors is same for different pair of communities. Here $k_{z}$ doesn't mean the degree of node $z$, but indicates the number of communities which have links with node $z$.

$$
C R\left(c_{i}, c_{j}\right)^{C R A A}=\sum_{z \in\left(\Gamma\left(c_{i}\right) \cup V\left(c_{i}\right)\right) \cap\left(\Gamma\left(c_{j}\right) \cup V\left(c_{j}\right)\right)} \frac{1}{\log k_{z}}
$$




$$
C R\left(c_{i}, c_{j}\right)^{C R R A}=\sum_{z \in\left(\Gamma\left(c_{i}\right) \cup V\left(c_{i}\right)\right) \cap\left(\Gamma\left(c_{j}\right) \cup V\left(c_{j}\right)\right)} \frac{1}{k_{z}}
$$

\section{Proposed algorithm}

There are three steps in this method. Firstly, extract the community structure by using the method Com_ST which only consider the local information of the network. Secondly, calculate the relevance of each pair of communities by using the new community relevance indices such as CRCN, CRJC, CRAA and CRRA. Finally, a simple prediction model which based on ruler inference is applied to estimate the probability of the missing links. The flow chart of our algorithm as follows: left side corresponding the processes of our algorithm and right side corresponding the techniques and methods we use.

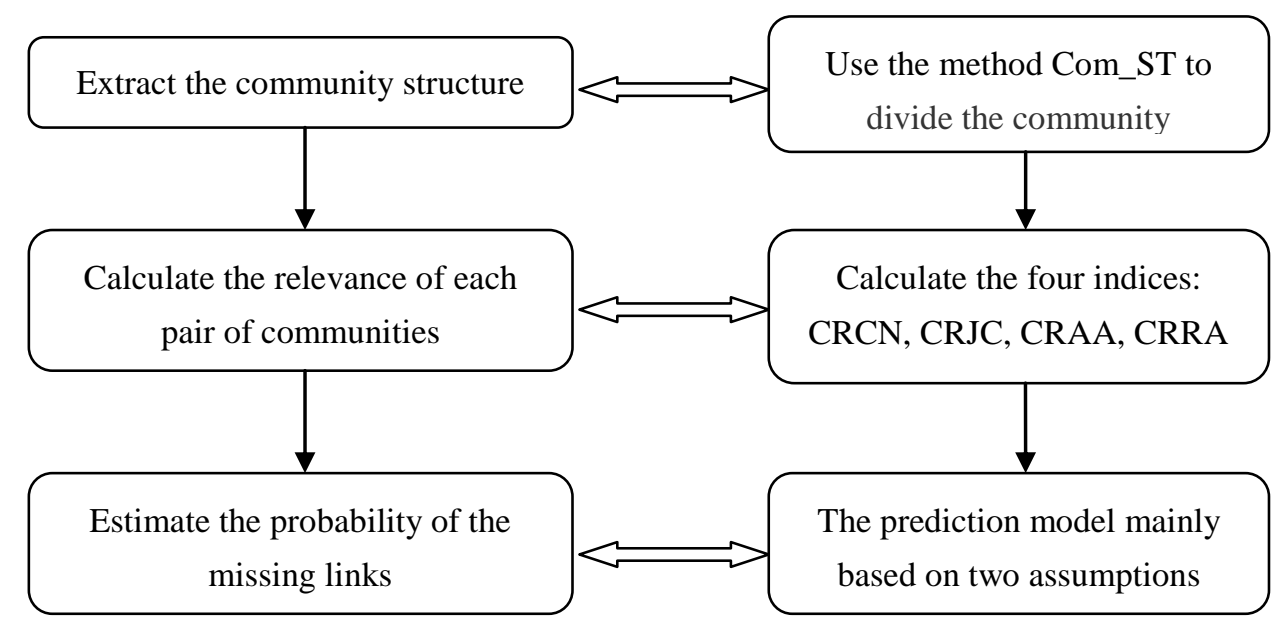

Fig.1. The flow chart of our algorithm

\subsection{Community Division}

Two steps are included in this algorithm: (1) Find the initial community; (2) Expand the community. Instead of the accurate division result obtained by a general community detection method, the proposed algorithm which was called Com_ST just needs the relatively accurate results obtained in the shortest possible time.

In the first step, we changed the method based on node strength in ref. [34] to find an initial community. The node strength in our paper is defined as follows:

$$
B\left(v, c_{i}\right)=\frac{d_{v}^{i n}\left(c_{i}\right)+1}{d_{v}\left(c_{i}\right)+1}
$$

Here, $d_{v}^{i n}\left(c_{i}\right)$ is the sum of the links from $v$ to nodes in $c_{i}$. While $d_{v}\left(c_{i}\right)$ express the degree of the node $v$. The node strength $B\left(v, c_{i}\right)$ is the ratio of the connections which connected to the nodes in community $c_{i}$ to all connections of the node $v$. This new node strength is fit to undirected network. 
In the second step, we proposed a novel gain function which named Community Link Density Increment (CLDI) to expand the community. The gain function is defined as follows:

$$
\Delta f\left(v, c_{i}\right)=\left.\frac{E\left(c_{i}\right)}{E\left(c_{i}\right)+L\left(c_{i}, \overline{c_{l}}\right)}\right|_{v \in c_{i}}-\left.\frac{E\left(c_{i}\right)}{E\left(c_{i}\right)+L\left(c_{i}, \overline{c_{l}}\right)}\right|_{v \notin c_{i}}
$$

Here, $E\left(c_{i}\right)$ is the sum of the edges inside community $c_{i}$, while $L\left(c_{i}, \bar{c}_{l}\right)$ is the sum of the edges incident to nodes in community $c_{i}$. The physical meaning of this function is to determine the influence on community link density when we put the node $v$ into the community $c_{i}$. Instead of the function $\mathrm{Q}$ which needs the global information of the network, this gain function just uses the local information, so the time complexity is low.

The detailed steps of the algorithm Com_ST are as follows:

Input: A network $G=G(V, E)$.

Output: An array of communities $C=\left\{c_{1}, c_{2}, \ldots, c_{m}\right\}$ of the network $G$

Step 1: Find the initial community

1) $\quad v=\max \left(d_{v}, v \in V\right)$ and $c_{0}=\{v, \Gamma(v)\}$; // Find the node with maximum degree and combine its neighbors to form a community $c_{0}$

2) Repeat: //Until for every $u \in \Gamma(v), B\left(u, c_{0}\right)>B^{H}$ is true.

For each $u \in \Gamma(v)$, do

If $B\left(u, c_{0}\right) \leq B^{H}, u \notin c_{0} ; / /$ We choose $\boldsymbol{B}^{\boldsymbol{H}}=\mathbf{0 . 5}$, if the node strength blow 0.5 , delete vertex $u$ from community $c_{0}$.

end

end

3) Output $\left(c_{0}\right)$; // Obtain the initial community $c_{0}$.

\section{Step 2: Expand the community}

1) Repeat: // Until for every $v \in \Gamma\left(c_{0}\right), B\left(v, c_{0}\right)<B^{L}$ is true.

for each $v \in \Gamma\left(c_{0}\right)$, do

if $B\left(v, c_{0}\right)>B^{H}, v \in c_{0}$;

else if $B\left(v, c_{0}\right)<B^{L}, v \notin c_{0} ; / /$ We choose $B^{L}=\mathbf{0 . 4}$, if the node strength blow 0.4 , delete vertex $v$ from community $c_{0}$.

else $v \in N(v)$;

end 


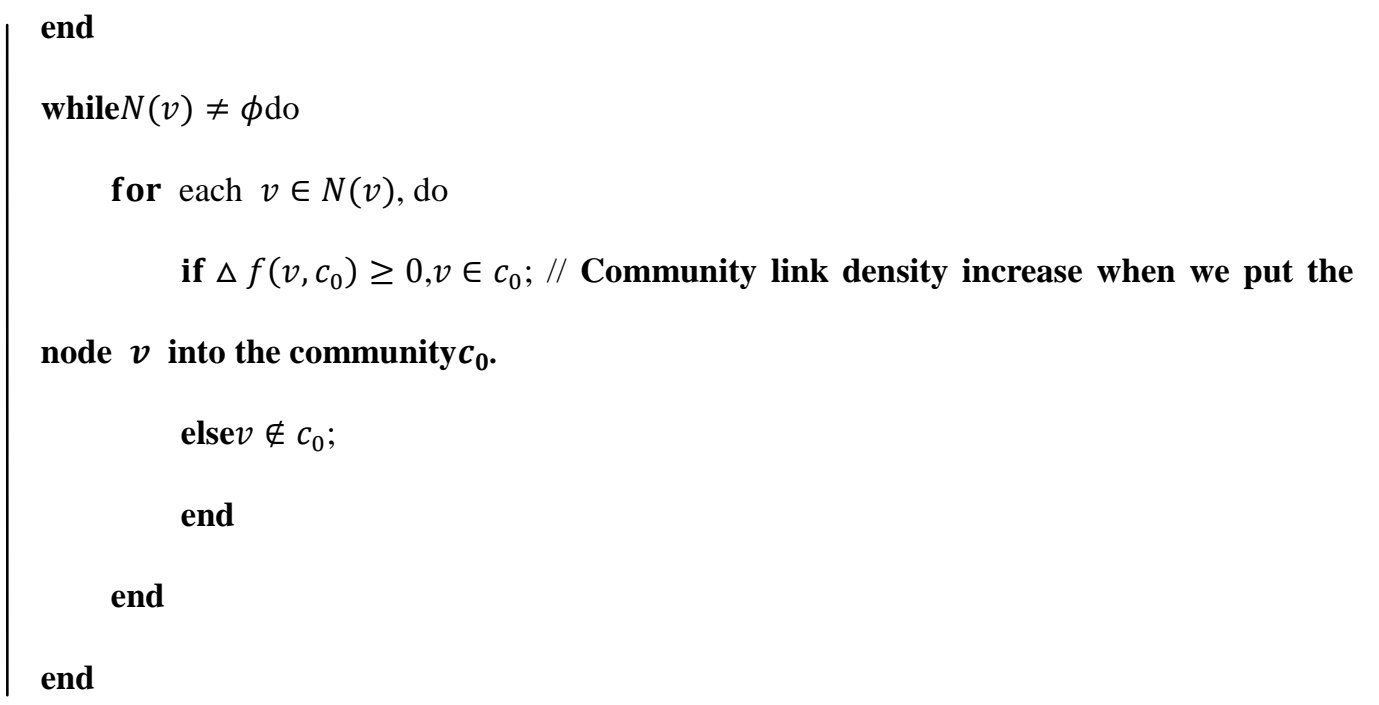

Fig.2. The pseudo codes of the algorithm Com_ST

Extract the community $c_{0}$, then repeat step 1 and step 2 for the remaining nodes until find out all communities. The illustration about the calculation of $B\left(v, c_{i}\right)$ and $\Delta f\left(v, c_{i}\right)$ can be found in Appendix A in the supplementary material.

\subsection{Community relevance}

At present, it's proved that the community structure information indeed provide insights for link prediction $[4,17]$. Almost all of the studies which using the community structure information to predict missing links just consider the assumption that links between the same community nodes are more similar and have higher existing probability than links between different communities. But no one has to fully tap the relationship between communities and research the effect of this relationship on link prediction. So in this paper we defined a community structure feature which is named community relevance. Community relevance is one of the most important characteristics of community structure information. In this paper, the community relevance values do not represent the importance of communities themselves. Instead, they are computed for each pair of communities which means that the level of closeness of two communities. In fact, the concrete form of community relevance can be varied from different perspectives. In this paper, we describe the community as a collection of nodes, so nodes similarity index for link prediction can be converted into the measurement to describe the relevance between communities. We can use the link prediction technology to extract the community relevance characteristics, as a new metric, the characteristics of community relevance we obtained can be used for link prediction again. Moreover, the complex network analysis and link prediction mechanisms are complementary [35]. For example, understanding the mechanism of link formation can 
help us predict missing links accurately and, in turn, accurate link prediction indeed gives evidence to some underlying mechanisms that drive network evolution [36]. The above idea is feasible.

As mentioned in the second paragraph of the introduction, more than a new index, our focus is on the new philosophy in the formulation of community-based indices. We advocate a shift in perspective from nodes to communities, and in particular from nodes similarity to community relevance. The specific definition of community relevance can be found in 2.2 .

\subsection{Prediction model}

We adopt the ruler inference to estimate the probability of missing links in the prediction model. Here, our reasoning mainly based on two assumptions as follows:

According to the definition of community, we believe that two nodes in the same community have much higher probability to generate a connection than two nodes in different communities [17]. So, we assume that if two nodes in the same community, the probability of the missing link between them is 1 , otherwise, the probability is directly proportional to the relevance of the communities which the two nodes belong to.

There are hub nodes in real network, in fact, the hub nodes with very high degree and it is easy to connect with other nodes. We called it rich-club phenomenon [37]. So we assume that if there is a new node, the probability of the missing link is proportional to the degree of the node which connected it.

The detail formula is as follows:

1) If $v_{x} \in c_{i}, v_{y} \in c_{j}, i, j \in\{1,2, \ldots, m\}$

$$
\begin{aligned}
& S_{v_{x} v_{y}}^{C R C N}= \begin{cases}1, & \text { if } i=j \\
C R\left(c_{i}, c_{j}\right)^{C R C N}, & \text { if } i \neq j\end{cases} \\
& S_{v_{x} v_{y}}^{C R J C}= \begin{cases}1, & \text { if } i=j \\
C R\left(c_{i}, c_{j}\right)^{C R J C}, & \text { if } i \neq j\end{cases} \\
& S_{v_{x} v_{y}}^{C R A A}= \begin{cases}1, & \text { if } i=j \\
C R\left(c_{i}, c_{j}\right)^{C R A A}, & \text { if } i \neq j\end{cases} \\
& S_{v_{x} v_{y}}^{C R R A}= \begin{cases}1, & \text { if } i=j \\
C R\left(c_{i}, c_{j}\right)^{C R R A}, & \text { if } i \neq j\end{cases}
\end{aligned}
$$

2) Else if $v_{x} \notin c_{i}, v_{y} \in c_{j}, i, j \in\{1,2, \ldots, m\}$, which means $v_{x}$ is a new node.

$$
S_{v_{x} v_{y}}=\frac{k_{v_{y}}}{|E|}
$$

3) Else if $v_{x} \in c_{i}, v_{y} \notin c_{j}, i, j \in\{1,2, \ldots, m\}$, which means $v_{y}$ is a new node.

$$
S_{v_{x} v_{y}}=\frac{k_{v_{x}}}{|E|}
$$

4) Else 


$$
S_{v_{x} v_{y}}=0
$$

Here, $k_{v_{x}}$ and $k_{v_{y}}$ indicate the degree of the node $v_{x}$ and $v_{y},|E|$ represents the number of observed edges.

\section{Experiments}

In this section, the proposed algorithm is evaluated from prediction accuracy, the time complexity and the universality by experiment on both computer-generated benchmark datasets and real-world datasets, and compared with ten well-known methods.

$\mathrm{BH}$ and $\mathrm{BL}$ are the empirical values we have chosen. In the experiment we found that the performance of our algorithm is not good enough when $\mathrm{BH}$ and $\mathrm{BL}$ are too big or too small. If $\mathrm{BH}$ is too large, too many single node communities are divided. This will greatly increase the time complexity of our algorithm; If BH is too small, all nodes may be divided in to the same cluster, so that we can't effectively extract the characteristics of the community relevance. After many attempts, the BH value is set to 0.5 .

\subsection{The comparison algorithms and the evaluation metric}

We compared with these methods: Common Neighbors (CN) [38], Jaccard Coefficient (JC) [39], Adamic-Adar index (AA) [40], Resource Allocation indeed (RA) [41], Hierarchical Structure (HSM) [9], Stochastic Block Model (SBM) [16], Structural Perturbation Method (SPM) [19] and CP [18]. Moreover, we compared with Cannistraci's method which based on the strategy of link-community [4]. Then, we compared with Yan's algorithm which based on the community structure [17].

The main idea of link prediction based on node similarity is that if the two nodes have a higher similarity value, a link between them has greater probability. Although the algorithm based on node similarity is relatively simple, it's still the mainstream research direction in academia. In this paper, we focus on four classical algorithms which based on node similarity. They are Common Neighbors (CN) [38], Jaccard Coefficient (JC) [39], Adar-Adamic (AA) [40] and Resource-Allocation (RA) [41]. Because the Resource-Allocation index of original is not good enough in stability, it was improved in ref. [1]. In this paper, we just compared with this new one. These node similarity algorithms defined as Table 2, where $\Gamma(x)$ denotes the set of neighbors of node $x, d_{z}$ denotes the degree of the node $z$.

\section{Table 2}

Four classical algorithms based on node similarity

\begin{tabular}{ll}
\hline \hline Node similarity algorithms & Scores \\
\hline
\end{tabular}




\begin{tabular}{cc}
\hline $\begin{array}{c}\text { Common Neighbors (CN) } \\
\text { Jaccard Index(JC) }\end{array}$ & $S_{x y}^{C N}=|\Gamma(x) \cap \Gamma(y)|$ \\
$S_{x y}^{J a c c a r d}$ & $=\frac{|\Gamma(x) \cap \Gamma(y)|}{|\Gamma(x) \cup \Gamma(y)|}$ \\
Adamic-Adar Index(AA) & $S_{x y}^{A A}=\sum_{z \in \Gamma(x) \cap \Gamma(y)} \frac{1}{\log d_{z}}$ \\
Resource-Allocation indeed(RA) & $S_{x y}^{R A}=\sum_{z \in \Gamma(x) \cap \Gamma(y)} \frac{1}{d_{z}}$ \\
\hline \hline
\end{tabular}

In addition to the above classic algorithms, we also compared with the method which proposed by Cannistraci [4]. His algorithm introduces a new philosophy in the formulation of neighborhood-based indices which is named CAR-based variant. CAR suggests that two nodes are more likely to link together if their common-first-neighbors are members of a strongly inner-linked cohort. The CAR-based neighborhood techniques defined as Table 3, where $\Gamma(x)$ denotes the set of neighbors of node $x, \gamma(z)$ refers to the sub-set of neighbors of $\mathrm{z}$ that are also common neighbors of $\mathrm{x}$ and $\mathrm{y}$, thus $\gamma(z)$ is the local community degree of $z$. These algorithms were taken as the comparison algorithms in subsequent experiments.

Table 3

The CAR-based neighborhood techniques

\begin{tabular}{cc}
\hline \hline Name of the index & Scores \\
\hline CAR & $S_{x y}^{C A R}=S_{x y}^{C N} \cdot \sum_{z \in \Gamma(x) \cap \Gamma(y)} \frac{|\gamma(z)|}{2}$ \\
CJC & $S_{x y}^{C J C}=\frac{S_{x y}^{C A R}}{|\Gamma(x) \cap \Gamma(y)|}$ \\
CAA & $S_{x y}^{C A A}=\sum_{z \in \Gamma(x) \cap \Gamma(y)} \frac{|\gamma(z)|}{\log _{2}(|\Gamma(z)|)}$ \\
CRA & $S_{x y}^{C R A}=\sum_{z \in \Gamma(x) \cap \Gamma(y)} \frac{|\gamma(z)|}{|\Gamma(z)|}$ \\
\hline \hline
\end{tabular}

In this paper, we adopt AUC, a standard metric, to quantify the accuracy of different link predicted methods [42]. In the present case, the AUC is an important performance measure which relates the sensitivity (true positive rate) and specificity (true negative rate) of a classifier [43]. In the implementation, among $n$ independent comparisons, if there are $n^{\prime}$ occurrences of the missing link having a higher score and $n^{\prime \prime}$ occurrences of the missing link and nonexistent link having the same score, the measurement AUC can be defined as

$$
A U C=\frac{n^{\prime}+0.5 n^{\prime \prime}}{n}
$$


This metric can be interpreted as the probability that a randomly chosen missing link (a link in $E^{P}$ ) is given a higher score than a randomly chosen nonexistent link (a link in $\widetilde{E}$, where $\widetilde{E}$ denotes the universal set except $E$ ). Thus, the value of degree exceeds $1 / 2$ indicates how much better the algorithm performs than pure chance.

\subsection{The datasets}

The Lancichinetti-Fortunato-Radicchl (LFR) benchmark was proposed to test the performance of algorithms for community detection [44, 45]. Here it is used to evaluate the performance of our proposed algorithm. We generate several un-weighted undirected benchmark networks when $N=100$ and 1000. For each $N$, six networks are generated with different parameters. The detailed parameters of the benchmark networks are given in Table 4. The parameters of the networks are given as follows: where $N$ is the number of nodes, $\langle k\rangle$ is the average degree of the nodes, $k_{\max }$ represents the maximum degree, $\gamma$ represents the exponent for the degree distribution, $\beta$ represents the exponent for the community size distribution, and $\mu$ is the mixing parameter which represents the ratio of the number of links between different communities to the total number of edges of the network. Here, $\mu$ is given six different values: $0.05,0.10,0.20,0.30,0.40$, and 0.50 , respectively. The larger the ratio is, the more difficult it is to reveal the community structure.

\section{Table 4}

Parameters of the networks

\begin{tabular}{ccccccc}
\hline \hline Dataset & $N$ & $\langle k\rangle$ & $k_{\max }$ & $\gamma$ & $\beta$ & $\mu$ \\
\hline 100 & 100 & 10 & 25 & 2 & 1 & From 0.05 to 0.50 \\
1000 & 1000 & 20 & 50 & 2 & 1 & From 0.05 to 0.50 \\
1000 & 1000 & 4 & 10 & 2 & 1 & From 0.05 to 0.50 \\
\hline \hline
\end{tabular}

Moreover, we tested sixteen different real-world networks in this section. These real networks for testing are college-football network (football), electrical power-grid of the western US (power), protein-protein interaction network (protein), co-authorship network of scientists who are themselves publishing on the topic of network science (netscience), Zachary's Karate Club network (karate), Bottlenose Dolphins network (dolphins), Jazz musicians network (jazz), Emails within an university network (email), co-appearance network of characters in the novel Les Miserables (lemis), network of the US air transportation system (USAir), network of grassland species interactions (grassland), network of terrorist association (terrorist), Amazon e-commerce network (amazon), network of hyperlinks between weblogs on US politics(polblogs), (sfi), (ssc), (Geom), (PGP). Most of them can be 
http://www-personal.umich.edu/ mejn/netdata/,http://deim.urv.cat/ alexandre.arenas/data/welcome.ht $\underline{m}$ and http://vlado.fmf.uni-lj.si/pub/networks/pajek/data/gphs.htm. The details of others can be found in ref. [46, 47].

\subsection{Compared with classical methods}

In this section, we mainly compared with CN, JC, AA, RA, HSM, SBM, SPM and CP. For each network we remove a subset of connections chosen uniformly at random as the probe set in the experiment. The probe sets range from $10 \%$ to $90 \%$. And then we attempt to predict missing links based on the remaining connections. For each network, we repeat the experiment 10 times. The final result is the average. Because the time cost of the methods HSM and SBM are too large, we can't obtain the result in acceptable time when $N$ above 1000. So we don't use our method to compare with HSM and SBM in the following experiments when $N$ above 1000. Fig. 3 plotted the AUC as a function of the fraction of observed edges in benchmark networks.

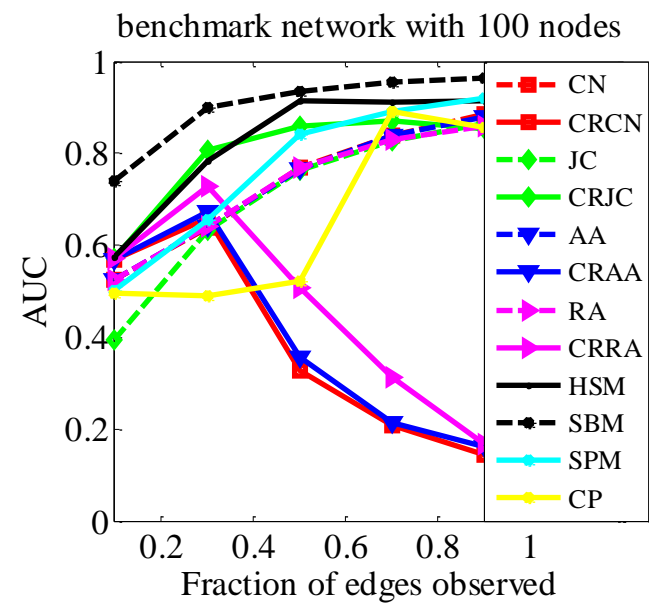

(a)

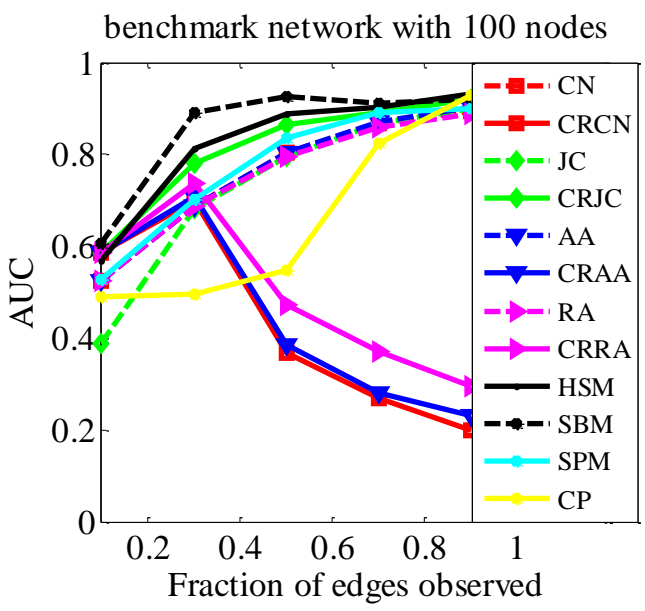

(b) 


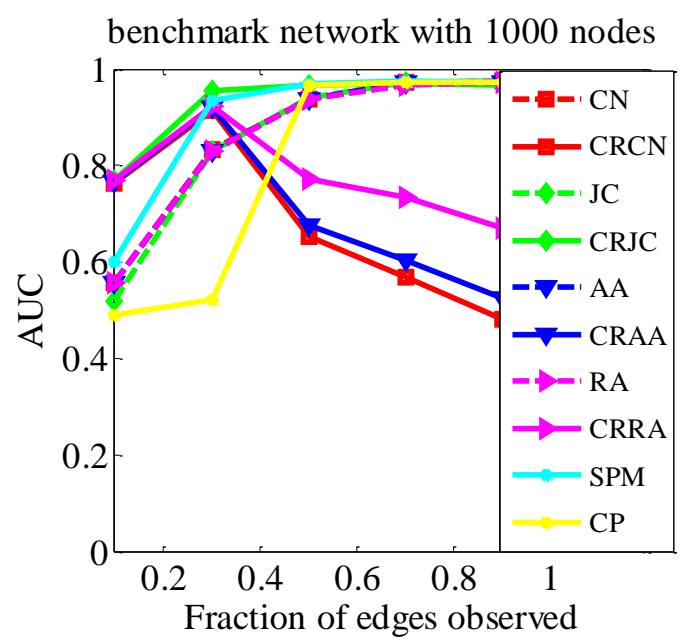

(c)

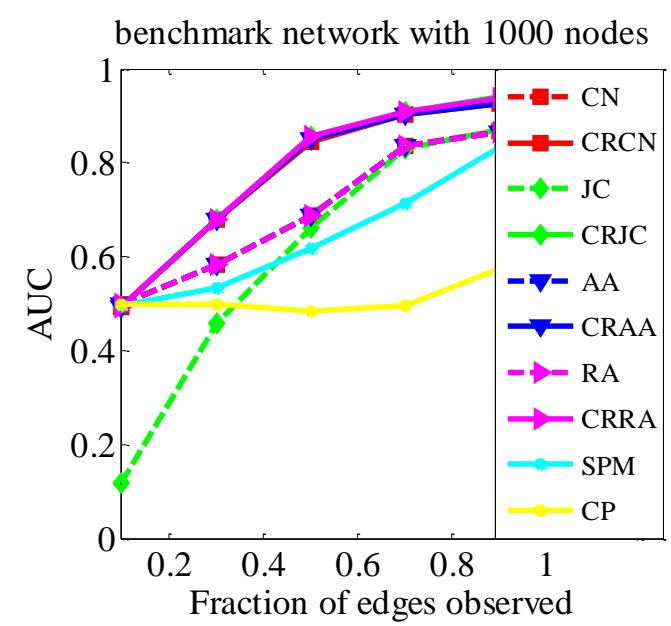

(e)

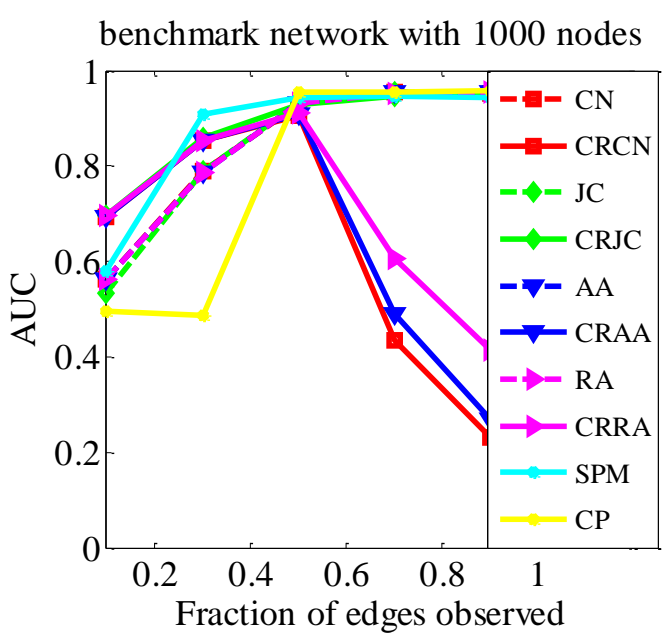

(d)

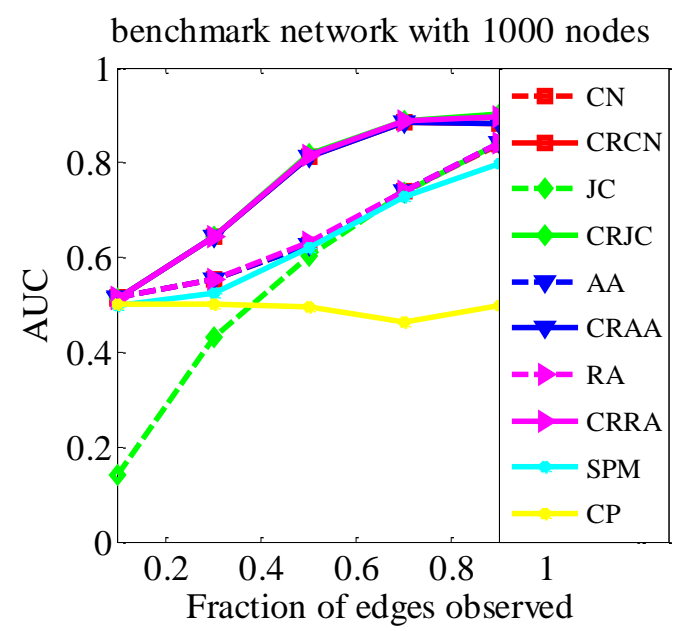

(f)

Fig.3. Plot the AUC as a function of the fraction of observed edges in benchmark networks. The lines with different colors indicate different methods, The red dashed line represents the $\mathrm{CN}$ algorithm; The red solid line indicates the $\mathrm{CRCN}$ algorithm; The green dashed line represents the JC algorithm; The green solid line represents the CRJC algorithm; The blue dashed line represents the AA algorithm; The blue solid line represents the CRAA algorithm; The rose red dashed line represents the RA algorithm; The rose red solid line represents the CRRA algorithm; The black solid line indicates the HSM algorithm; The black dashed line indicates the SBM algorithm; The cyan solid line indicates the SPM algorithm; The yellow solid line indicates the $\mathrm{CP}$ algorithm. (a) $N=100, \mu=0.05,<k>=10$ and $k_{\text {max }}=25$. (b) $N=100, \mu=0.10,<k>=10$ and $k_{\max }=25$. (c) $N=1000, \mu=0.05,<k>=20$ and $k_{\text {max }}=50$. (d) $N=1000, \mu=0.10,<k>=20$ and $k_{\text {max }}=50$. (e) $N=1000, \mu=0.05,<k>=4$ and $k_{\max }=10$. (f) $N=1000, \mu=0.10,<k>=4$ and $k_{\max }=10$.

Our algorithm performs better than other methods except HSM and SBM, but the time complexity 
of ours is much lower than theirs. The results indicate that under the premise of ensuring the prediction accuracy, our algorithm with low time complexity. From Fig. 3 (a), (b), (c), (d), we can see that the performance of our algorithm is better than other traditional similarity algorithms when the proportion of observations less than $30 \%$. The results indicate that under the same time complexity, our algorithm with higher prediction accuracy. However, the performance curve will decline when the proportion of observations more than $30 \%$. This is not happened in (e), (f). Compared to the networks of (a), (b), (c), (d), the networks of (e), (f) has the characteristic of degree distribution consistency. That means under different proportions of the observations, the degree distributions of the networks almost have no change (Appendix B in the Supplementary Material). This property ensures the stability of our algorithm in the first step of community division.

Moreover, our algorithm performs better than other similarity methods when the observed edges are very few even in the networks which are not consistent in degree distribution. That means our algorithm suits the network with more destroyed. Next, we tested the prediction accuracy of our proposed algorithm when the proportion of observed edges is $10 \%$ and 30\%. Table 5, Table 6 show the results when $N=100,<k>=10, k_{\max }=25$ and $N=1000,<k>=20, k_{\max }=50$.

\section{Table 5}

Show the AUC predicted by twelve algorithms for each benchmark network when the proportion of observed edges is $10 \%$ and $30 \%$. Here $N=100,<k>=10, k_{\max }=25$.

\begin{tabular}{|c|c|c|c|c|c|c|c|c|c|c|c|c|}
\hline & \multicolumn{2}{|c|}{0.05} & \multicolumn{2}{|c|}{0.10} & \multicolumn{2}{|c|}{0.20} & \multicolumn{2}{|c|}{0.30} & \multicolumn{2}{|c|}{0.40} & \multicolumn{2}{|c|}{0.50} \\
\hline algorithns & $10 \%$ & $30 \%$ & $10 \%$ & $30 \%$ & $10 \%$ & $30 \%$ & $10 \%$ & $30 \%$ & $10 \%$ & $30 \%$ & $10 \%$ & $30 \%$ \\
\hline $\mathrm{CN}$ & 0.525 & 0.639 & 0.525 & 0.687 & 0.530 & 0.625 & 0.513 & 0.554 & 0.508 & 0.552 & 0.506 & 0.545 \\
\hline CRCN & 0.564 & 0.745 & 0.598 & 0.728 & 0.569 & 0.671 & 0.539 & 0.581 & 0.522 & 0.554 & 0.513 & 0.539 \\
\hline $\mathrm{JC}$ & 0.395 & 0.632 & 0.390 & 0.681 & 0.385 & 0.606 & 0.372 & 0.541 & 0.365 & 0.539 & 0.394 & 0.541 \\
\hline CRJC & 0.57 & 0.787 & 0.601 & 0.763 & 0.570 & 0.703 & 0.539 & 0.591 & 0.521 & 0.557 & 0.513 & 0.539 \\
\hline AA & 0.525 & 0.642 & 0.525 & 0.687 & 0.530 & 0.628 & 0.513 & 0.556 & 0.508 & 0.551 & 0.506 & 0.543 \\
\hline CRAA & 0.564 & 0.748 & 0.599 & 0.730 & 0.569 & 0.677 & 0.539 & 0.586 & 0.522 & 0.554 & 0.513 & 0.541 \\
\hline RA & 0.525 & 0.642 & 0.525 & 0.687 & 0.530 & 0.629 & 0.513 & 0.556 & 0.508 & 0.551 & 0.506 & 0.543 \\
\hline CRRA & 0.565 & 0.761 & 0.600 & 0.741 & 0.569 & 0.689 & 0.539 & 0.589 & 0.521 & 0.551 & 0.513 & 0.540 \\
\hline HSM & 0.576 & 0.783 & 0.568 & 0.812 & 0.583 & 0.694 & 0.561 & 0.591 & 0.545 & 0.585 & 0.564 & 0.614 \\
\hline SBM & 0.74 & 0.9 & 0.605 & 0.89 & 0.535 & 0.765 & 0.485 & 0.65 & 0.635 & 0.595 & 0.565 & 0.605 \\
\hline SPM & 0.501 & 0.655 & 0.527 & 0.703 & 0.500 & 0.672 & 0.500 & 0.575 & 0.493 & 0.552 & 0.507 & 0.541 \\
\hline $\mathrm{CP}$ & 0.497 & 0.491 & 0.489 & 0.497 & 0.501 & 0.479 & 0.501 & 0.482 & 0.477 & 0.513 & 0.508 & 0.507 \\
\hline
\end{tabular}

Table 6

Show the AUC predicted by ten algorithms for each benchmark network when the proportion of 
observed edges is $10 \%$ and $30 \%$. Here $N=1000,<k>=20, k_{\max }=50$.

\begin{tabular}{|c|c|c|c|c|c|c|c|c|c|c|c|c|}
\hline & \multicolumn{2}{|c|}{0.05} & \multicolumn{2}{|c|}{0.10} & \multicolumn{2}{|c|}{0.20} & \multicolumn{2}{|c|}{0.30} & \multicolumn{2}{|c|}{0.40} & \multicolumn{2}{|c|}{0.50} \\
\hline algorithy & $10 \%$ & $30 \%$ & $10 \%$ & $30 \%$ & $10 \%$ & $30 \%$ & $10 \%$ & $30 \%$ & $10 \%$ & $30 \%$ & $10 \%$ & $30 \%$ \\
\hline $\mathrm{CN}$ & 0.558 & 0.833 & 0.563 & 0.789 & 0.551 & 0.733 & 0.526 & 0.656 & 0.521 & 0.634 & 0.518 & 0.579 \\
\hline CRCN & 0.753 & 0.908 & 0.693 & 0.854 & 0.618 & 0.757 & 0.555 & 0.710 & 0.55 & 0.658 & 0.498 & 0.593 \\
\hline $\mathrm{JC}$ & 0.52 & 0.831 & 0.534 & 0.79 & 0.533 & 0.733 & 0.491 & 0.658 & 0.496 & 0.638 & 0.494 & 0.579 \\
\hline CRJC & 0.752 & 0.911 & 0.695 & 0.859 & 0.619 & 0.759 & 0.557 & 0.711 & 0.55 & 0.658 & 0.498 & 0.592 \\
\hline AA & 0.558 & 0.829 & 0.564 & 0.788 & 0.551 & 0.734 & 0.526 & 0.66 & 0.521 & 0.634 & 0.518 & 0.581 \\
\hline CRAA & 0.753 & 0.908 & 0.694 & 0854 & 0.618 & 0.757 & 0.553 & 0.711 & 0.55 & 0.659 & 0.498 & 0.593 \\
\hline RA & 0.558 & 0.829 & 0.564 & 0788 & 0.551 & 0.734 & 0.526 & 0.662 & 0.521 & 0.634 & 0.518 & 0.581 \\
\hline CRRA & 0.752 & 0.906 & 0.695 & 0.854 & 0.618 & 0.757 & 0.553 & 0.710 & 0.55 & 0.659 & 0.498 & 0.593 \\
\hline SPM & 0.601 & 0.934 & 0.581 & 0.909 & 0.572 & 0.845 & 0.549 & 0.789 & 0.529 & 0.724 & 0.514 & 0.66 \\
\hline $\mathrm{CP}$ & 0.491 & 0.521 & 0.495 & 0.488 & 0.486 & 0.465 & 0.481 & 0.533 & 0.474 & 0.53 & 0.494 & 0.458 \\
\hline
\end{tabular}

Table 5 and Table 6 analyze the advantages of our algorithm quantitatively when the observed edges are very few. From above tables, we can see that our method is more efficient than other node similarity algorithms when the fraction of observed edges is small. That's because the reduction of the training set will directly affect the accuracy of the neighborhood indices seriously, but the impact on the community relevance indices was not so great.

Next, we show some experiment results in real-world networks. The details are shown in Fig.4.

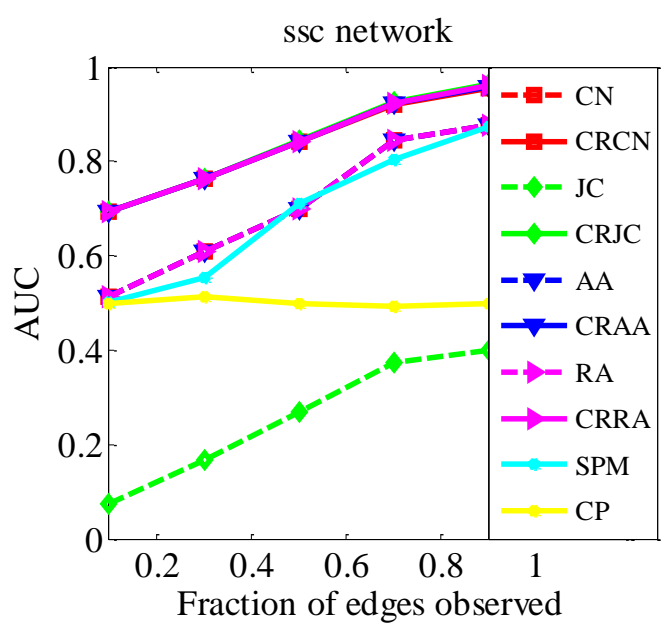

(a)

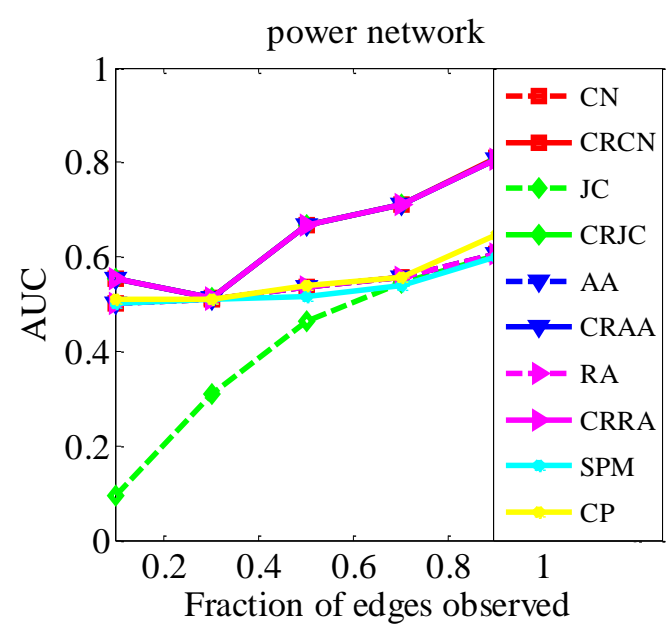

(b) 


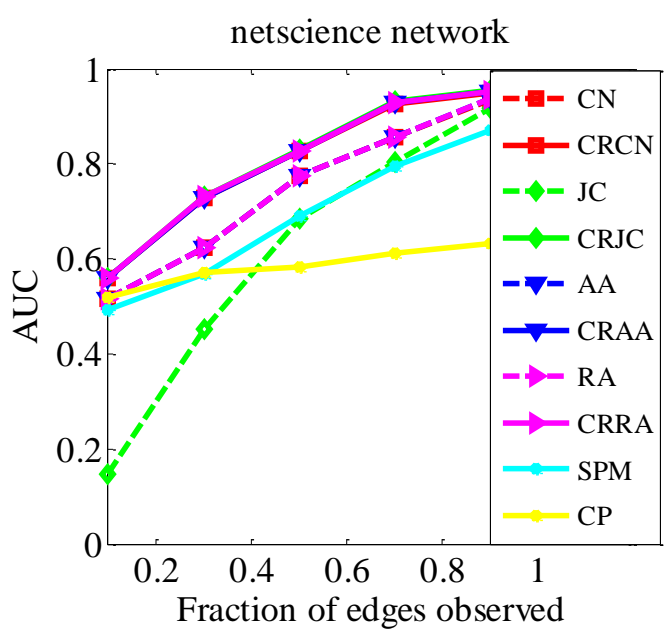

(c)

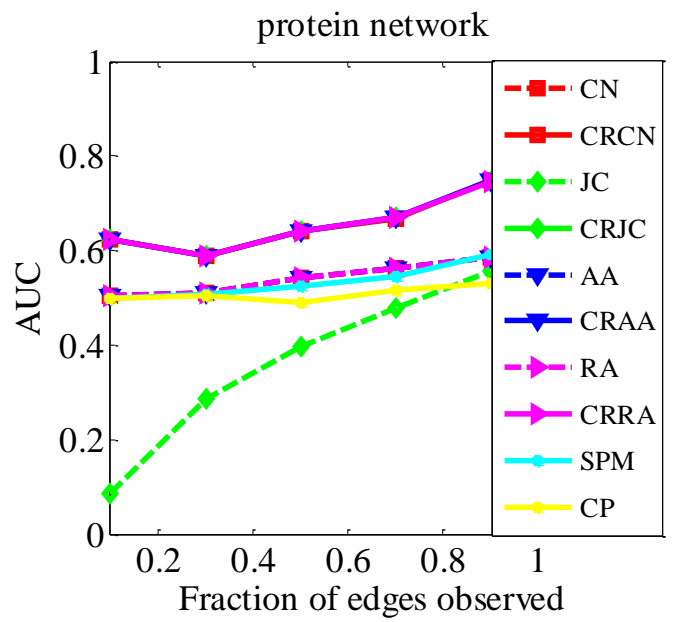

(e)

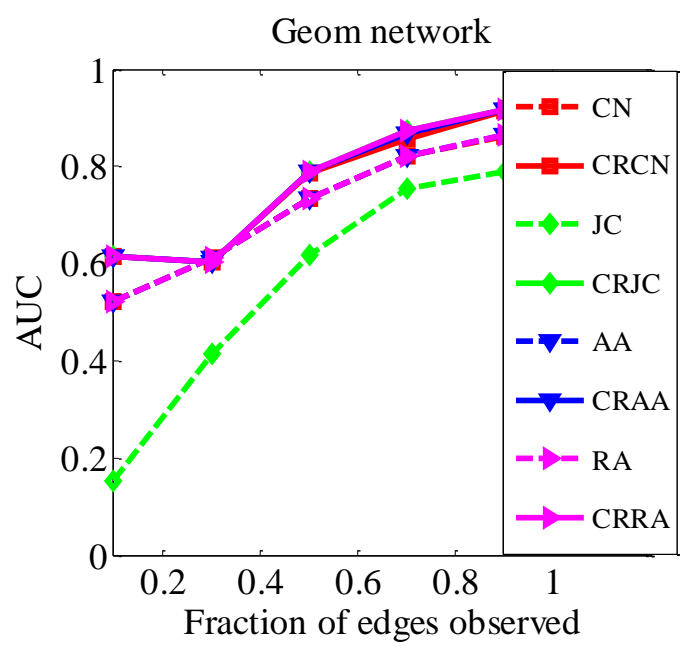

(g)

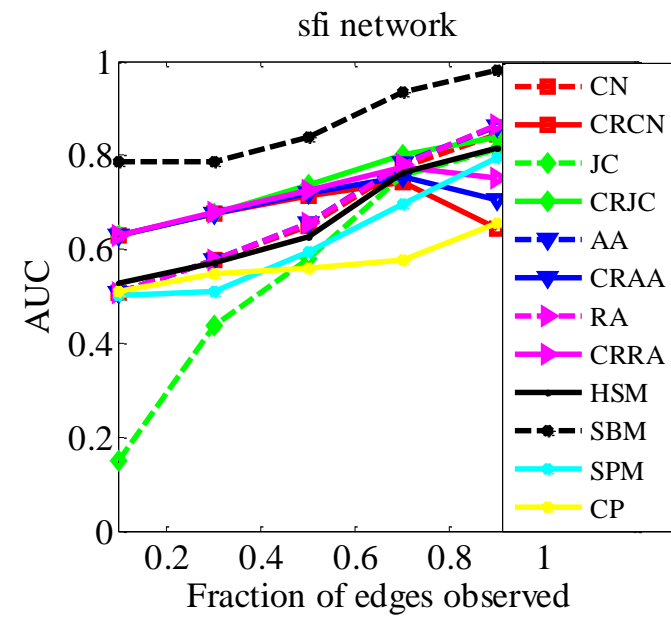

(d)

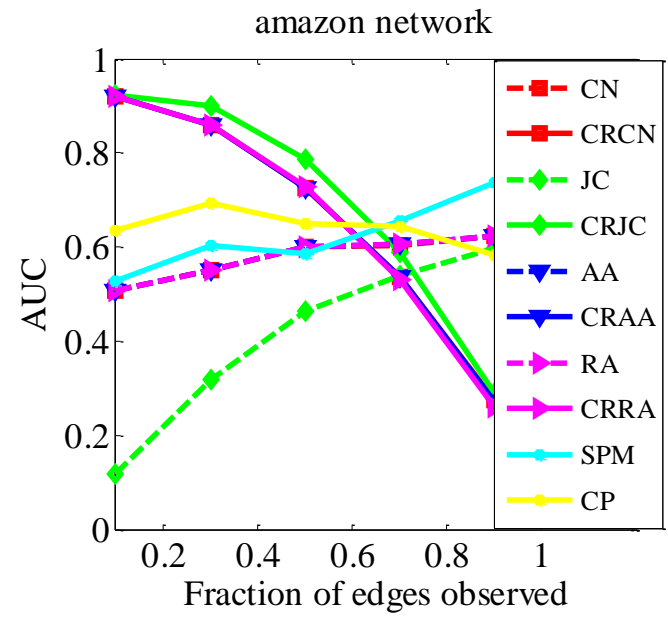

(f)

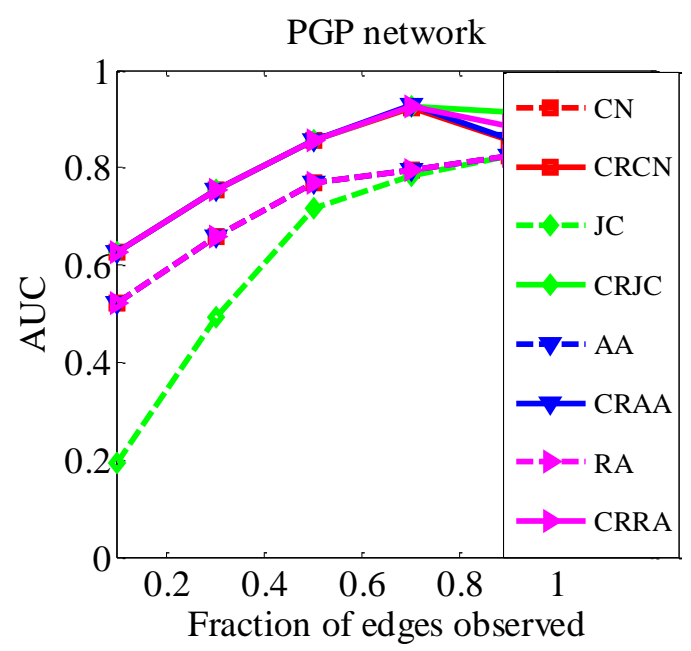

(h)

Fig.4. Plot the AUC as a function of the fraction of observed edges in real-world networks. (a) Show the results of ssc network. Here, $N=961$ and $E=1192$. (b) Show the results of electrical power-grid of the western US. Here, $N=4941$ and $E=13188$. (c) Show the results of co-authorship network of 
scientists. Here, $N=1589$ and $\mathrm{E}=5484$. (d) Show the results of sfi network. Here, $N=118$ and $\mathrm{E}=400$. (e) Show the results of protein-protein interaction network. Here, $N=2112$ and $E=4406$. (f) Show the results of Amazon e-commerce network. Here, $N=2879$ and E=7772. (g) Show the results of Geom network. Here, $N=7343$ and $\mathrm{E}=23796$. (h) Show the results of PGP network. Here, $N=10680$ and $\mathrm{E}=48632$. In each subfigure the $\mathrm{X}$ axis represents the proportion of observed edges, and $\mathrm{Y}$ axis represents AUC. The lines with different colors indicate different methods. The red dashed line represents the $\mathrm{CN}$ algorithm; The red solid line indicates the $\mathrm{CRCN}$ algorithm; The green dashed line represents the JC algorithm; The green solid line represents the CRJC algorithm; The blue dashed line represents the AA algorithm; The blue solid line represents the CRAA algorithm; The rose red dashed line represents the RA algorithm; The rose red solid line represents the CRRA algorithm; The black solid line indicates the HSM algorithm; The black dashed solid line indicates the SBM algorithm; The cyan solid line indicates the SPM algorithm; The yellow solid line indicates the CP algorithm.

Above two figures proved that our method works well in the real-world networks. Then, we test the time consumption of our algorithm and compared it with HSM, SBM, CP and SPM. The result showed in Fig. 5.

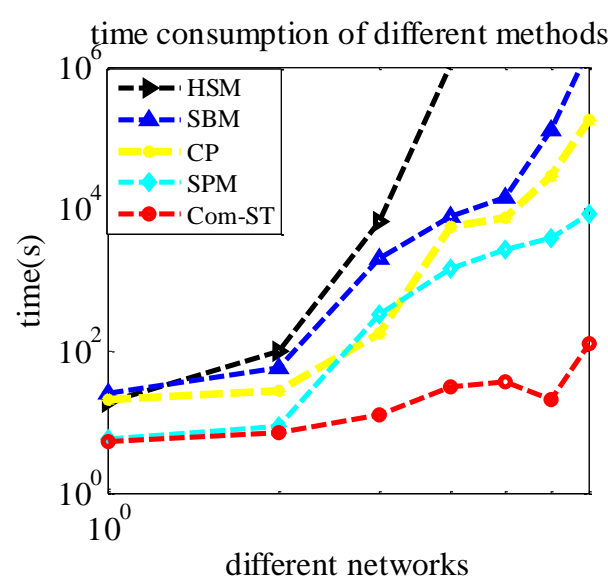

Fig.5. Plot the time cost of the five algorithms in different size of networks. X axis represents different networks. The coordinates on $\mathrm{X}$ axis represents karate, football, ssc, netscience, protein, amazon and power respectively. Y axis represents the time consumption. The lines with different colors indicate different methods.

In time consumption, our algorithm has obvious advantage. Here, the equipment and environment in our experiment are the same. The specific configuration is as follows, CPU: Intel(R) Core(TM) i5-3470; Operating system: win7; Host frequency: $3.2 \mathrm{GHz}, \mathrm{RAM}$ : 4G. Now we calculate the mean 
prediction accuracy for all comparison methods. And give the rank of the prediction accuracy of our methods in table 8 . Here we just consider the benchmark networks which $N=1000$ and $<k>=4$. First, we list some characteristic parameters of the network in table 7. The characteristic parameters of the networks which used in the experiments are as follows: $N$ is the number of nodes in the network; $E$ is the number of edges, $Q$ is the modularity, $\langle k\rangle$ is the average degree of the nodes, $C$ is the clustering coefficient and $d$ is the average path length of the network.

Table 7

Parameters of the networks

\begin{tabular}{|c|c|c|c|c|c|c|}
\hline Network & $N$ & $E$ & $Q$ & $<k>$ & C & $d$ \\
\hline Karate & 34 & 156 & 0.381 & 4.588 & 0.571 & 2.408 \\
\hline terrorist & 62 & 304 & 0.500 & 4.903 & 0.486 & 2.946 \\
\hline dolphins & 62 & 318 & 0.495 & 5.129 & 0.259 & 3.357 \\
\hline lemis & 77 & 508 & 0.501 & 6.597 & 0.573 & 2.641 \\
\hline grassland & 86 & 226 & 0.659 & 3.013 & 0.295 & inf \\
\hline football & 115 & 1226 & 0.577 & 10.66 & 0.403 & 2.508 \\
\hline sfi & 118 & 400 & 0.733 & 3.390 & 0.612 & 5.021 \\
\hline jazz & 198 & 5484 & 0.439 & 27.70 & 0.618 & 2.235 \\
\hline USAirline & 332 & 4252 & 0.320 & 12.81 & 0.625 & 2.738 \\
\hline ssc & 961 & 1192 & 0.695 & 4.550 & 0.132 & inf \\
\hline email & 1133 & 10902 & 0.512 & 9.622 & 0.220 & 3.606 \\
\hline polblogs & 1490 & 33430 & 0.427 & 27.31 & 0.263 & $\inf$ \\
\hline netscience & 1589 & 5484 & 0.956 & 3.754 & 0.638 & $\inf$ \\
\hline protein & 2112 & 4406 & 0.845 & 2.387 & 0.060 & inf \\
\hline amazon & 2879 & 7772 & 0.669 & 2.700 & 0.074 & 3.433 \\
\hline power & 4941 & 13188 & 0.934 & 2.669 & 0.080 & 18.989 \\
\hline Geom & 7343 & 23796 & 0.773 & 3.864 & 0.408 & inf \\
\hline PGP & 10680 & 48632 & 0.852 & 4.554 & 0.266 & 7.486 \\
\hline 100_0.05 & 100 & 948 & 0.677 & 9.48 & 0.558 & 2.752 \\
\hline 100_0.10 & 100 & 992 & 0.64 & 9.92 & 0.584 & 2.753 \\
\hline $100 \_0.20$ & 100 & 966 & 0.609 & 9.66 & 0.516 & 2.489 \\
\hline 100_0.30 & 100 & 920 & 0.471 & 9.2 & 0.273 & 2.411 \\
\hline 100_0.40 & 100 & 1036 & 0.312 & 10.36 & 0.238 & 2.249 \\
\hline 100_0.50 & 100 & 994 & 0.307 & 9.94 & 0.201 & 2.26 \\
\hline 1000_0.05 & 1000 & 3556 & 0.949 & 3.556 & 0.608 & $\inf$ \\
\hline 1000_0.10 & 1000 & 3586 & 0.924 & 3.586 & 0.537 & $\inf$ \\
\hline 1000_0.20 & 1000 & 3536 & 0.857 & 3.536 & 0.403 & inf \\
\hline 1000_0.30 & 1000 & 3560 & 0.799 & 3.56 & 0.297 & $\inf$ \\
\hline 1000_0.40 & 1000 & 3498 & 0.711 & 3.498 & 0.151 & $\inf$ \\
\hline 1000_0.50 & 1000 & 3632 & 0.634 & 3.632 & 0.075 & 5.633 \\
\hline
\end{tabular}

Table 8 
The rank of the prediction accuracy of our methods

\begin{tabular}{ccccc}
\hline \hline Network & CRCN & CRJC & CRAA & CRRA \\
\hline Karate & 11 & 5 & 10 & 8 \\
terrorist & 12 & 5 & 10 & 9 \\
dolphins & 10 & $\mathbf{3}$ & 9 & 7 \\
lemis & 11 & 6 & 10 & 9 \\
grassland & 6 & $\mathbf{2}$ & $\mathbf{4}$ & $\mathbf{3}$ \\
football & 12 & $\mathbf{4}$ & 11 & 10 \\
sfi & 5 & $\mathbf{2}$ & $\mathbf{4}$ & $\mathbf{3}$ \\
jazz & 11 & 8 & 10 & 9 \\
USAirline & 11 & 7 & 9 & 8 \\
ssc & $\mathbf{4}$ & $\mathbf{1}$ & $\mathbf{3}$ & $\mathbf{2}$ \\
email & $\mathbf{3}$ & $\mathbf{2}$ & $\mathbf{3}$ & 5 \\
polblogs & 6 & 8 & 5 & 6 \\
netscience & $\mathbf{4}$ & $\mathbf{1}$ & $\mathbf{3}$ & $\mathbf{2}$ \\
protein & $\mathbf{4}$ & $\mathbf{1}$ & $\mathbf{3}$ & $\mathbf{2}$ \\
amazon & $\mathbf{2}$ & $\mathbf{1}$ & $\mathbf{3}$ & $\mathbf{4}$ \\
power & $\mathbf{2}$ & $\mathbf{1}$ & $\mathbf{2}$ & $\mathbf{2}$ \\
Geom & $\mathbf{4}$ & $\mathbf{1}$ & $\mathbf{3}$ & $\mathbf{1}$ \\
PGP & $\mathbf{4}$ & $\mathbf{1}$ & $\mathbf{3}$ & $\mathbf{2}$ \\
$100 \_0.05$ & 12 & $\mathbf{3}$ & 11 & 9 \\
$100 \_0.10$ & 12 & $\mathbf{3}$ & 11 & 10 \\
$100 \_0.20$ & 12 & $\mathbf{4}$ & 11 & 10 \\
$100 \_0.30$ & 12 & $\mathbf{3}$ & 9 & 8 \\
$100 \_0.40$ & 10 & 6 & 9 & 8 \\
$100 \_0.50$ & 8 & 9 & 6 & 7 \\
$1000 \_0.05$ & $\mathbf{4}$ & $\mathbf{1}$ & $\mathbf{3}$ & $\mathbf{2}$ \\
1000_0.10 & $\mathbf{4}$ & $\mathbf{1}$ & $\mathbf{3}$ & $\mathbf{2}$ \\
1000_0.20 & $\mathbf{4}$ & $\mathbf{1}$ & $\mathbf{3}$ & $\mathbf{2}$ \\
1000_0.30 & $\mathbf{4}$ & $\mathbf{1}$ & $\mathbf{3}$ & $\mathbf{2}$ \\
1000_0.40 & $\mathbf{4}$ & $\mathbf{1}$ & $\mathbf{2}$ & $\mathbf{2}$ \\
$1000 \_0.50$ & $\mathbf{4}$ & $\mathbf{1}$ & $\mathbf{3}$ & $\mathbf{2}$ \\
\hline \hline
\end{tabular}

Table 8 showed the rank of the prediction precision of our methods. And we can find that our method is more applicable to the networks which have obvious community structure. Moreover, the degree distributions are consistent. The characteristic of degree distribution consistency may ensure the stability of our algorithm in the first step of community division. It's because that if the degree distributions of the network are not consistent, the structure of the network is not stable enough. The network may have inconsistent information which will influence the community division (Appendix B in the Supplementary Material). Inaccurate community structure information will lead to the poor performance of our prediction method. We evaluate this finding with statistical methods. (Appendix C 
in the Supplementary Material). Compared these four community relevance indices, CRJC performs the best, and CRCN performs the worst, that because when the pairs of communities have the equal CRCN, it's not necessary for them to got the same CRJC. That is to say, the effective solutions of CRJC are much more than CRCN's (Appendix D in the Supplementary Material). Analyze from Table 7 and Table 8, we found that the bigger the modularity is, the better the performance of the algorithm will be. From the result, the critical value of the modularity is 0.5 .

\subsection{Compared with Cannistraci's algorithm}

In this section, we want to compare our method with Cannistraci's algorithm which based on the strategy of link-community. Fig. 6 showed the compared results both in benchmark networks and real-world networks.

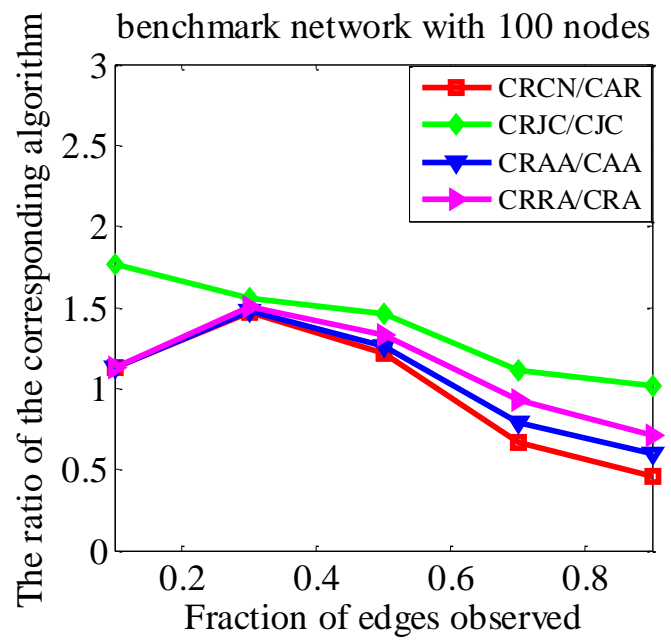

(a)

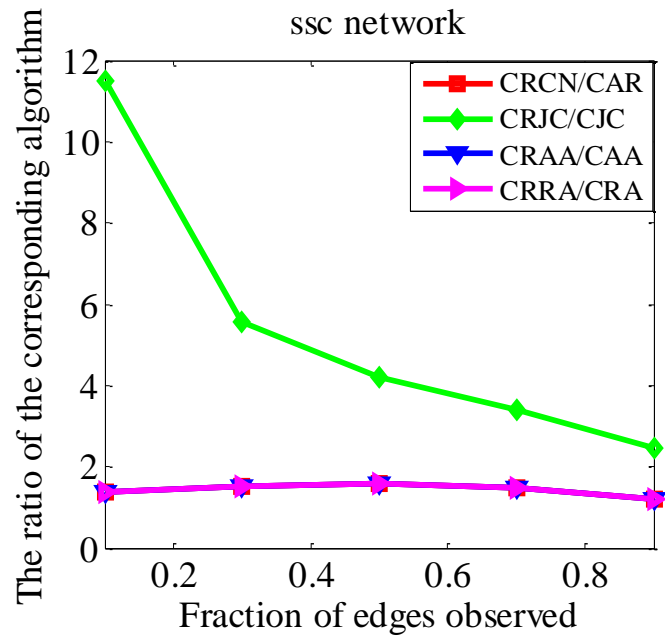

(c)

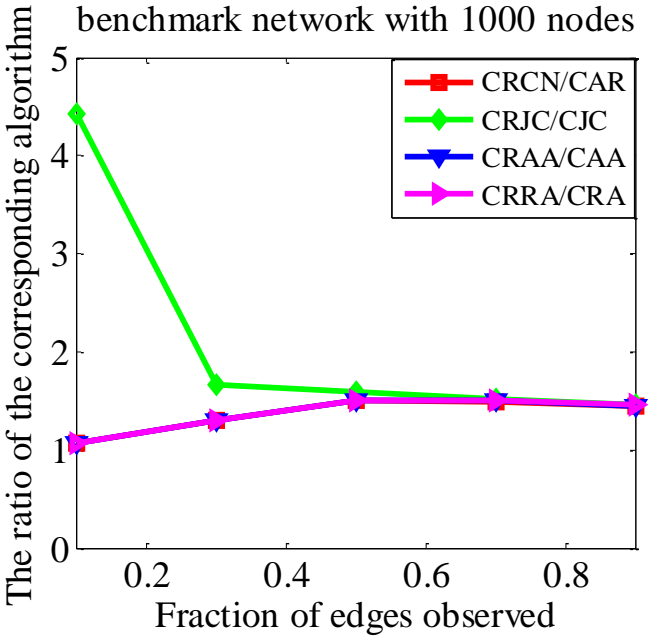

(b)

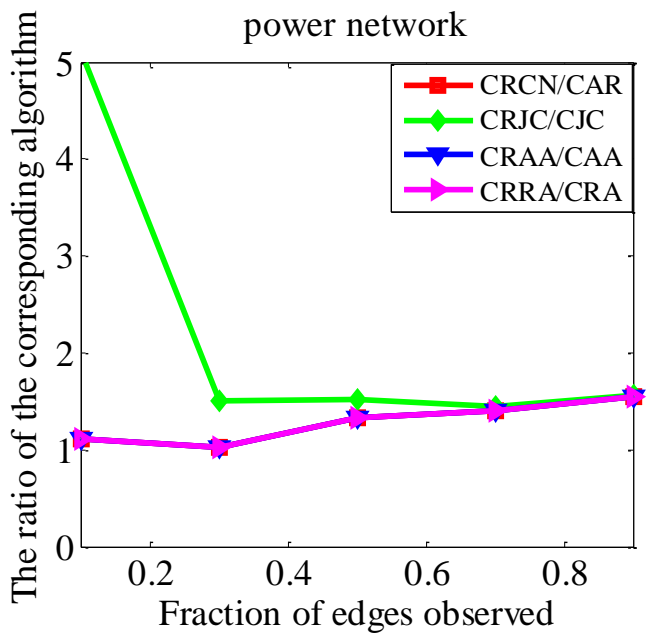

(d) 


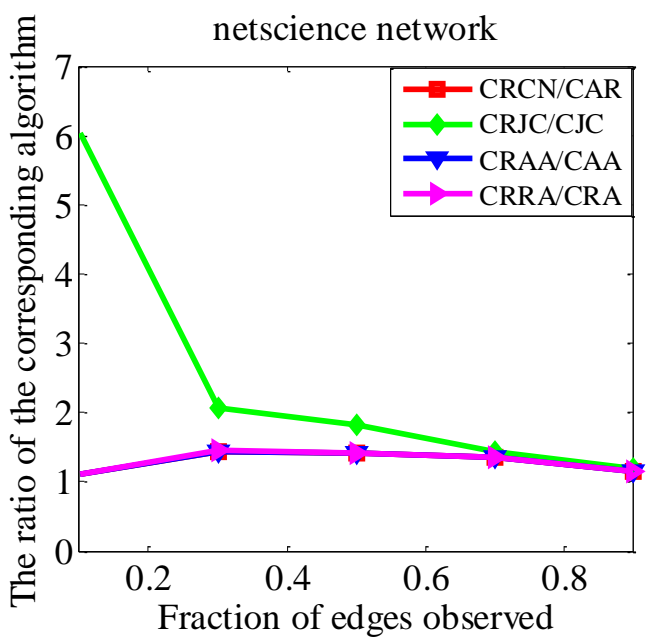

(e)

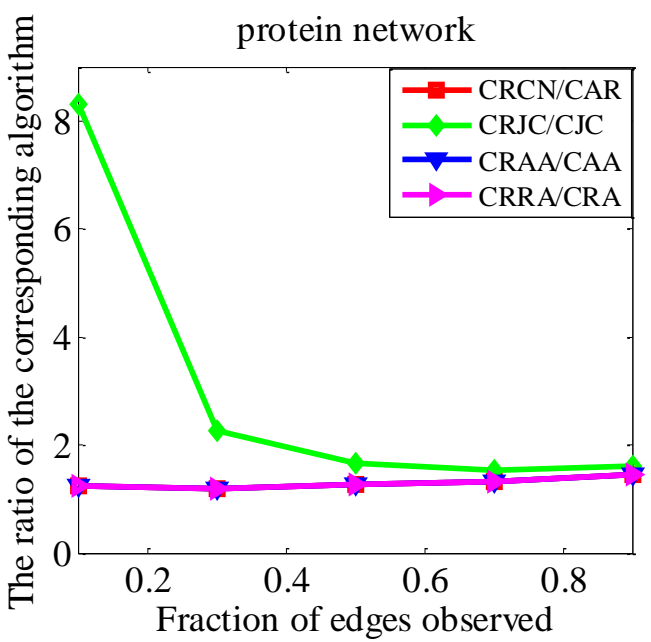

(g)

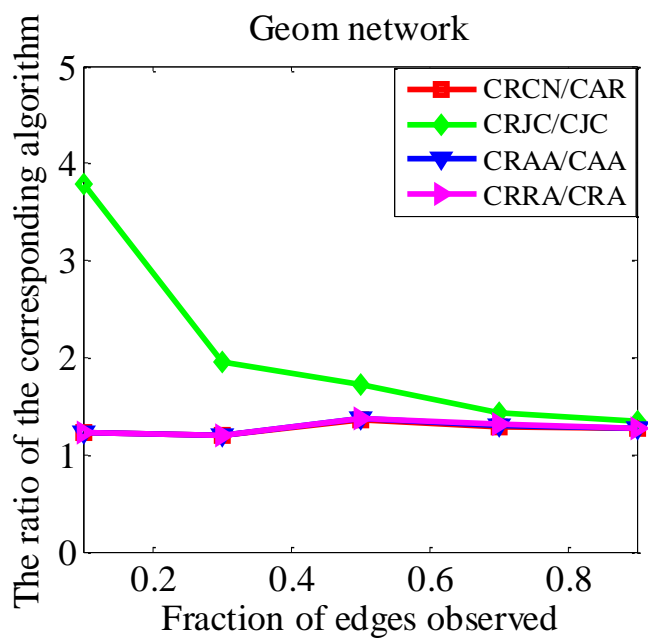

(i)

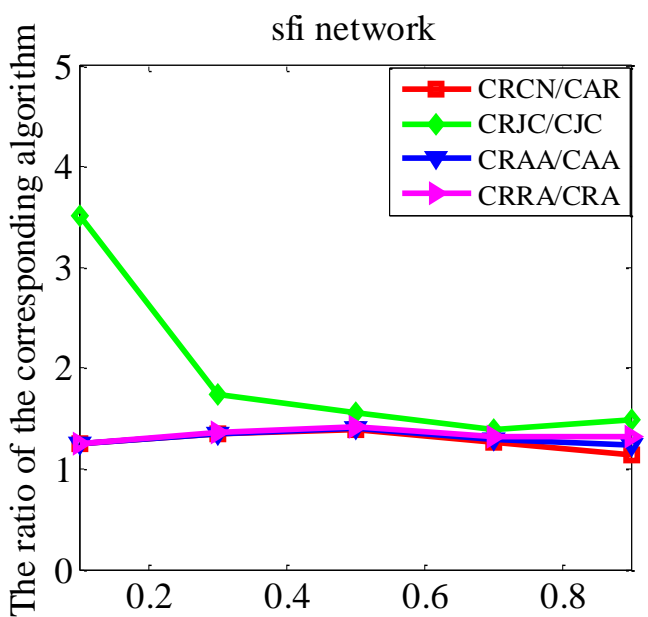

(f)

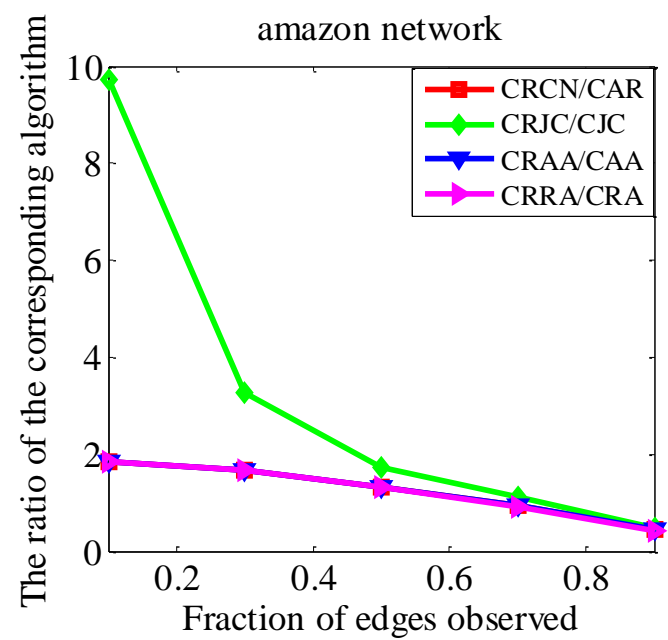

(h)

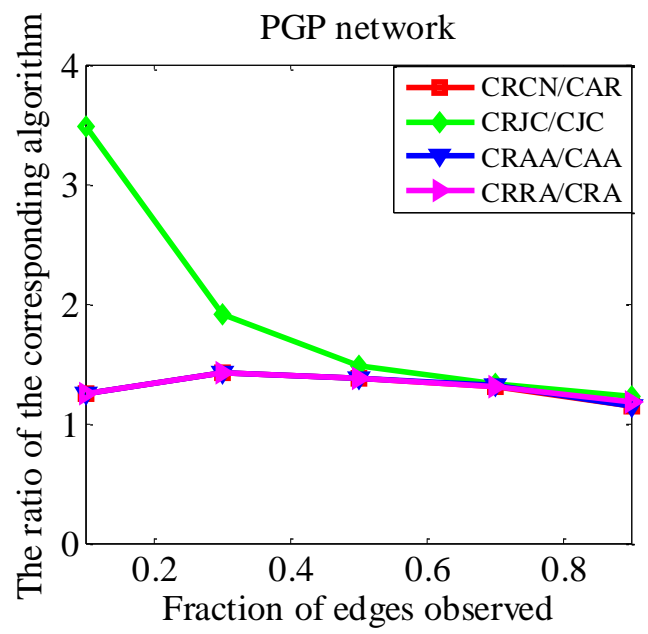

(j)

Fig.6. Plot the ratio of the AUC which predicted by our algorithm and Cannistraci's algorithm respectively. (a), (b) show the results of benchmark network. Here, (a) $N=100, \mu=0.05,<k>=10$ and $k_{\text {max }}=25$. (b) $N=1000, \mu=0.05,<k>=4$ and $k_{\max }=10$. (c), (d), (e), (f), (g), (h), (i), (j) show the 
results of real-world networks. In each subfigure the $\mathrm{X}$ axis represents the proportion of observed edges. The lines with different colors indicate different methods.

Fig. 6 shows the comparison results of our algorithm and Cannistraci's algorithm, $\mathrm{X}$ axis represents observation proportion, $\mathrm{Y}$ axis is the ratio of the AUC predicted by our algorithm to the AUC predicted by Cannistraci's algorithm. Now we calculate the mean values of the ratio of the AUC predicted by different algorithms under different proportions of observations. And give them in table 9. The value of degree exceeds 1 indicates how much better the proposed algorithm performs than Cannistraci’s algorithm.

Table 9

The mean values of the ratio of the AUC predicted by different algorithms under different proportions of observations

\begin{tabular}{|c|c|c|c|c|}
\hline \multirow{2}{*}{$\begin{array}{c}\text { Network } \\
\text { Karate }\end{array}$} & \multicolumn{4}{|c|}{ CRCN/CARCRJC/CJCCRAA/CAACRRA/CRA } \\
\hline & 1.0496 & 1.5601 & 1.0535 & 1.0808 \\
\hline terrorist & 1.0358 & 1.5229 & 1.0579 & 1.0863 \\
\hline dolphins & 1.1020 & 1.4256 & 1.1063 & 1.1342 \\
\hline lemis & 0.98474 & 1.4308 & 1.0152 & 1.0652 \\
\hline grassland & 1.2407 & 2.6056 & 1.2536 & 1.2760 \\
\hline football & 0.9825 & 1.2189 & 1.0035 & 1.0504 \\
\hline sfi & 1.2798 & 1.9337 & 1.3086 & 1.3344 \\
\hline jazz & 0.9768 & 1.0611 & 0.9835 & 0.9857 \\
\hline USAirline & 1.0556 & 1.2388 & 1.0662 & 1.0756 \\
\hline ssc & 1.4350 & 5.4225 & 1.4365 & 1.4376 \\
\hline email & 1.2058 & 1.3440 & 1.2057 & 1.2033 \\
\hline polblogs & 1.1118 & 1.2376 & 1.1151 & 1.1106 \\
\hline netscience & 1.2948 & 2.5106 & 1.2962 & 1.2970 \\
\hline protein & 1.2926 & 3.0753 & 1.2940 & 1.2931 \\
\hline amazon & 1.2453 & 3.2592 & 1.2427 & 1.2377 \\
\hline power & 1.2829 & 2.2364 & 1.2819 & 1.2819 \\
\hline Geom & 1.2694 & 2.0484 & 1.2740 & 1.2755 \\
\hline PGP & 1.2420 & 1.0390 & 1.2420 & 1.2420 \\
\hline 100_0.05 & 0.9883 & 1.3814 & 1.0489 & 1.1210 \\
\hline $100 \_0.10$ & 0.9229 & 1.4309 & 0.9457 & 0.9938 \\
\hline 100_0.20 & 0.8317 & 1.2716 & 0.8463 & 0.9054 \\
\hline $100 \_0.30$ & 1.0728 & 1.2503 & 1.0901 & 1.1061 \\
\hline 100_0.40 & 1.0890 & 1.1979 & 1.0950 & 1.0975 \\
\hline 100_0.50 & 1.0881 & 1.1665 & 1.0911 & 1.0907 \\
\hline 1000_0.05 & 1.3608 & 2.1310 & 1.3617 & 1.3648 \\
\hline 1000_0.10 & 1.3569 & 2.0810 & 1.3577 & 1.3636 \\
\hline 1000_0.20 & 1.3216 & 2.0616 & 1.3224 & 1.3271 \\
\hline
\end{tabular}




\begin{tabular}{lllll}
$1000 \_0.30$ & 1.2840 & 2.1298 & 1.2866 & 1.2916 \\
$1000 \_0.40$ & 1.1912 & 1.8371 & 1.1922 & 1.1922 \\
$1000 \_0.50$ & 1.1220 & 1.7491 & 1.1230 & 1.1231 \\
\hline \hline
\end{tabular}

From the table 9, we found that our algorithm is better than Cannistraci's algorithm, especially the CRJC index.

\subsection{The universality of our algorithm}

Our algorithm is mainly composed of three parts. The first part is the community division which cost much time. In order to verify the first part of our algorithm is universal which means whatever the community detection method we choose, the result we obtained has very little influence on the subsequent operations. Except Com_ST, we also use the famous algorithm CNM [48] which proposed by Newman for the first step. The predicted results are shown in Fig. 7:

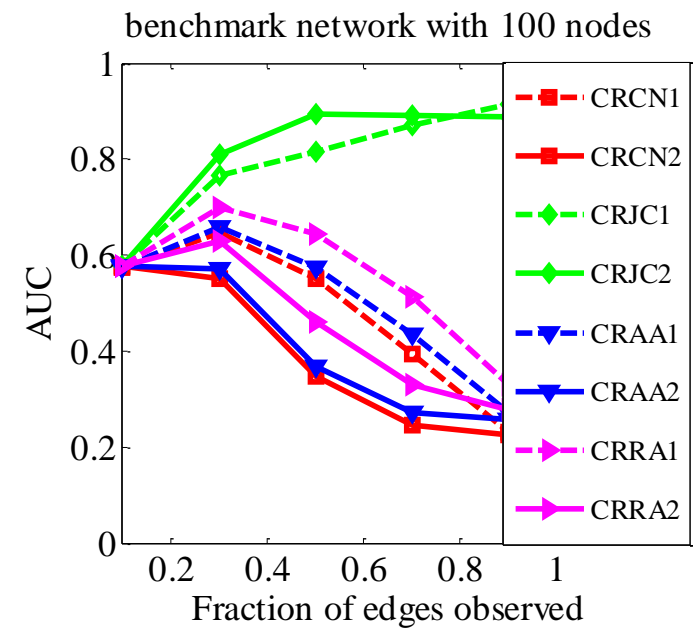

(a)

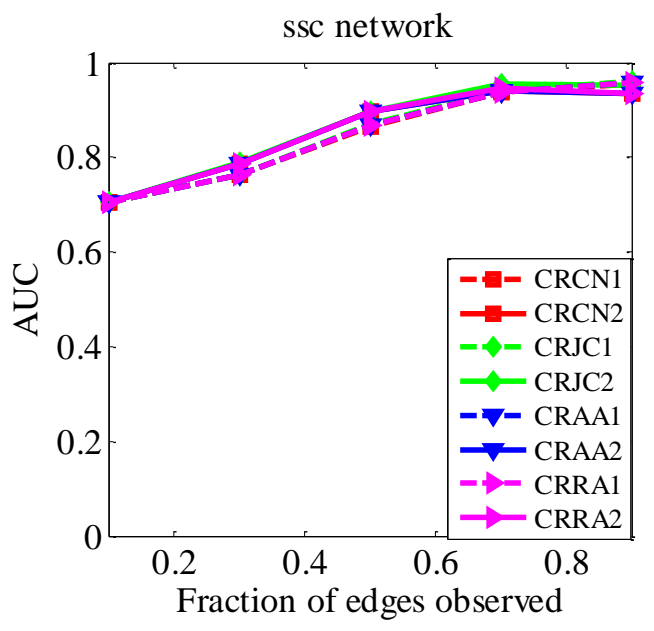

(c)

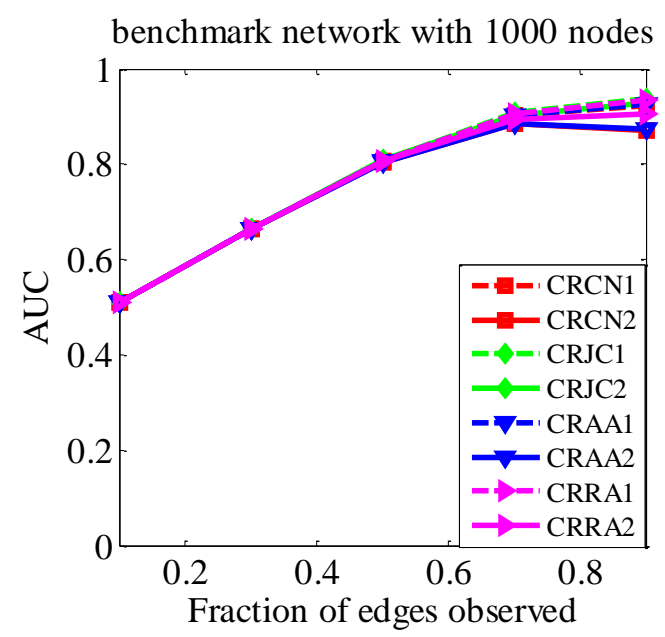

(b)

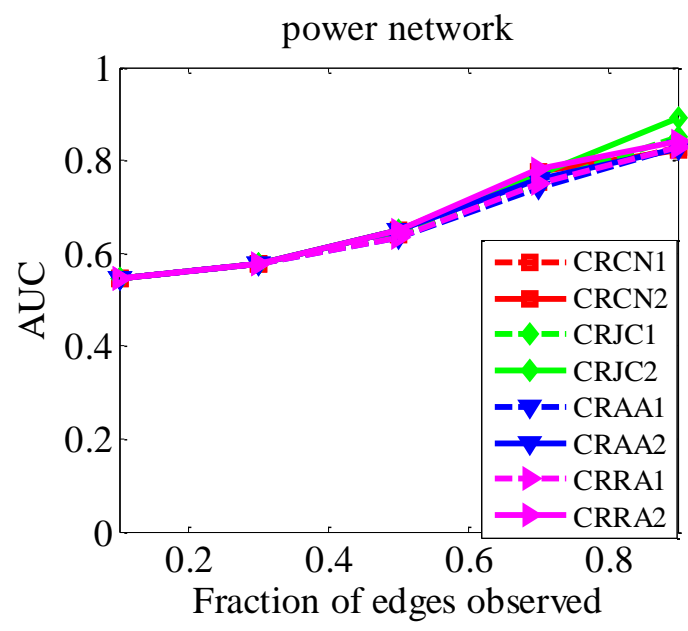

(d) 


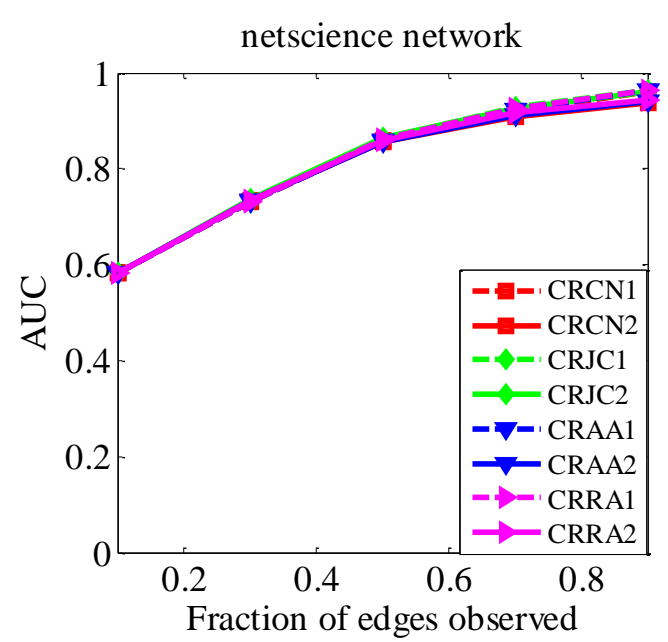

(e)

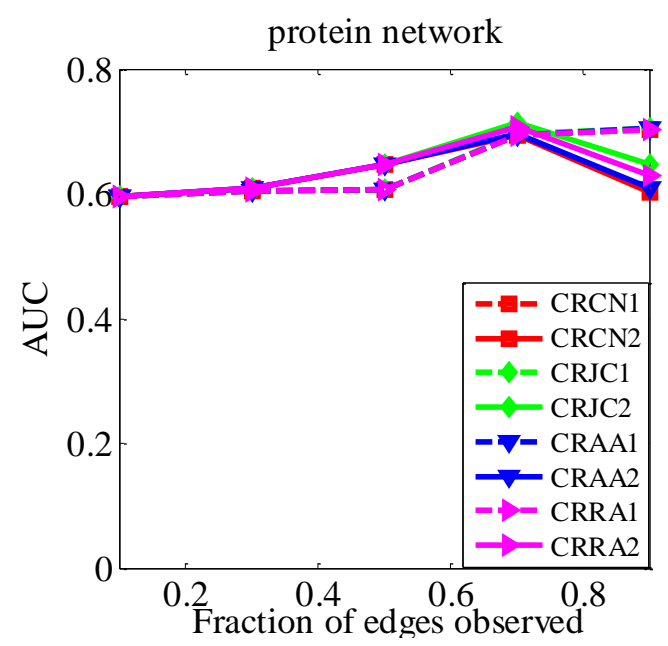

(g)

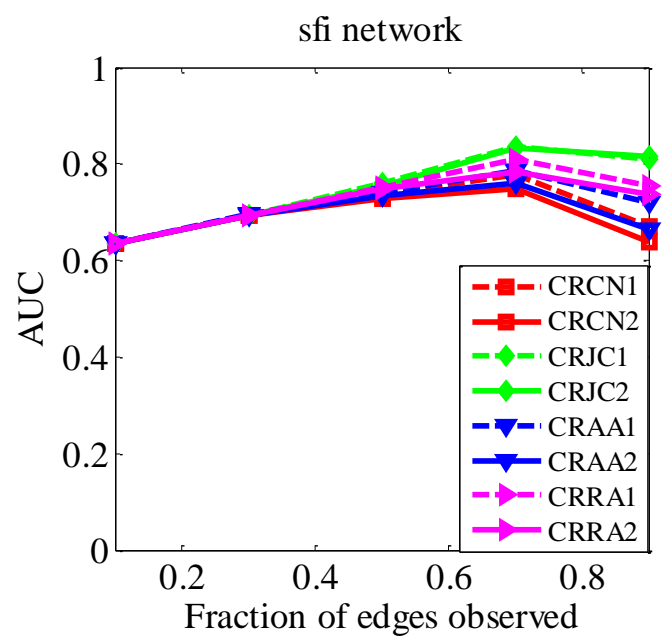

(f)

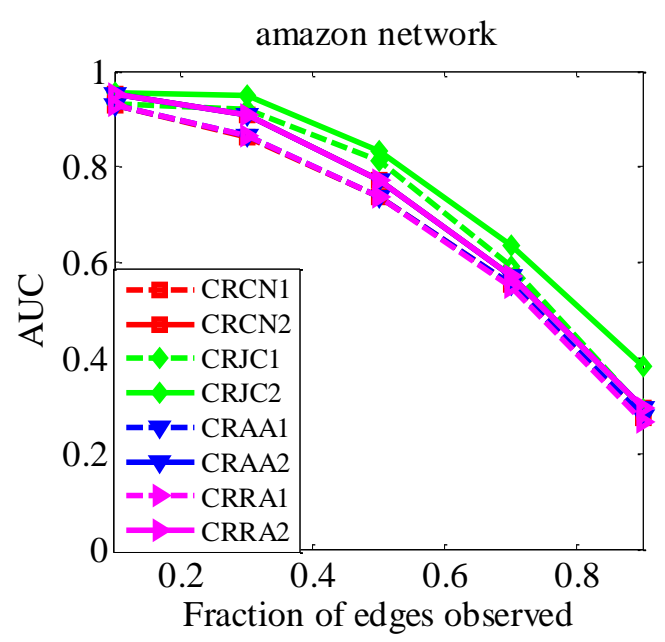

(h)

Fig.7. Plot the prediction results with different community division methods. (a), (b) Show the results of benchmark network. Here, (a) $N=100, \mu=0.05,\langle k\rangle=10$ and $k_{\max }=25$. (b) $N=1000$, $\mu=0.05,<k>=4$ and $k_{\max }=10$. (c), (d), (e), (f), (g), (h) Show the results of real-world networks. The lines with different colors indicate different community division methods we use. The red dashed line represents the CRCN1 algorithm; The red solid line indicates the CRCN2 algorithm; The green dashed line represents the CRJC1 algorithm; The green solid line represents the CRJC2 algorithm; The blue dashed line represents the CRAA1 algorithm; The blue solid line represents the CRAA2 algorithm; The rose red dashed line represents the CRRA1 algorithm; The rose red solid line represents the CRRA2 algorithm; CRCN1, CRJC1, CRAA1, CRRA1 indicate that the community division algorithm is Com_ST. CRCN2, CRJC2, CRAA2, CRRA2 indicate that the community division algorithm is CNM. In each subfigure the $\mathrm{X}$ axis represents the proportion of observed edges.

Otherwise, we test the time consumption of Com_ST and CNM. The result showed in Fig. 8. 


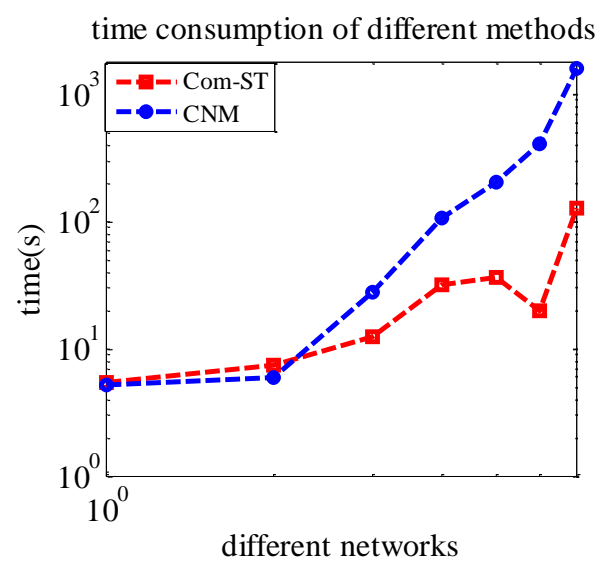

Fig.8. Plot the time cost of different algorithms. X axis represents different networks. The coordinates on $\mathrm{X}$ axis represents karate, football, ssc, netscience, protein, amazon and power respectively. $\mathrm{Y}$ axis represents the time consumption. The lines with different colors indicate different methods.

It can be seen in the chart that the performance of our algorithm is not sensitive to the algorithm which we use for the first step. That is to say, we can choose some low time complexity algorithm for the first process. This can reduce the time consumption of our algorithm.

\subsection{Compared with Yan's algorithm}

Because the performance of our algorithm is not sensitive to the detection algorithm which we use, so it has good universality. Next we want to test the performance of our method and compared with Yan's algorithm by using the same community detection method with low time complexity. The results are show in fig. 9

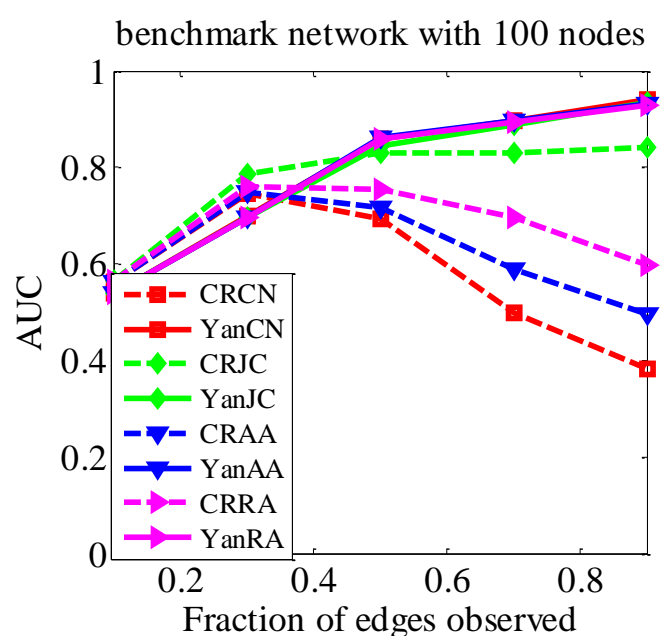

(a)

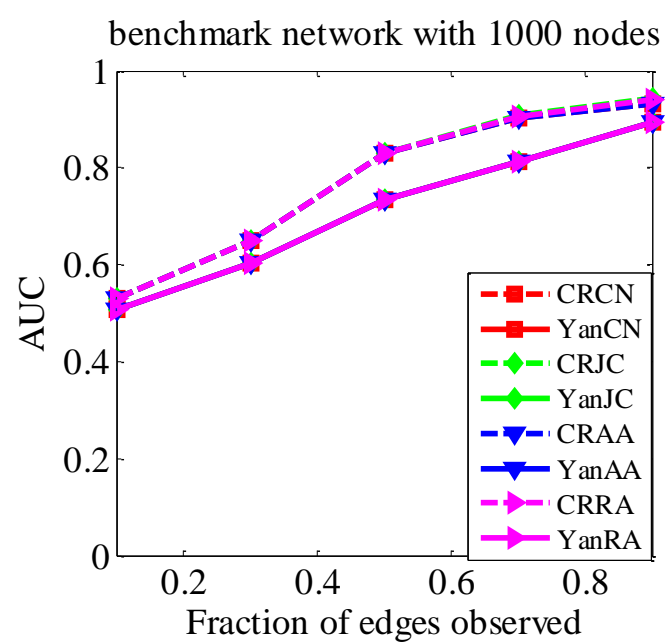

(b) 


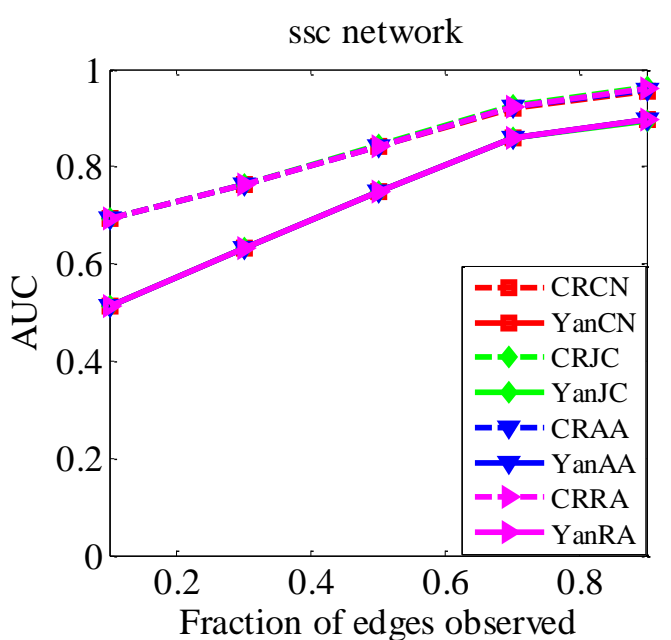

(c)

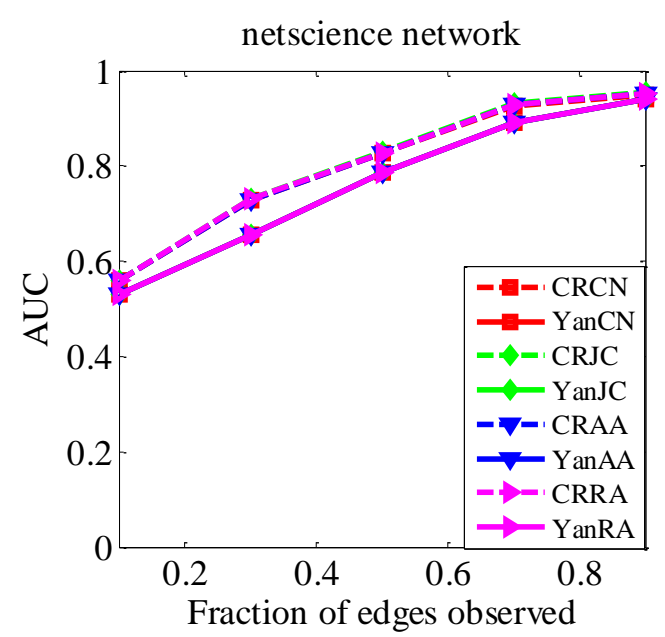

(e)

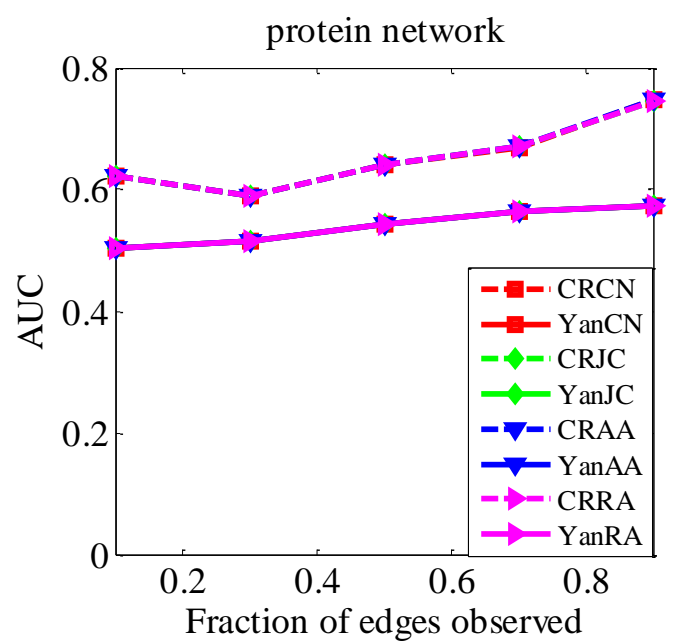

(g)

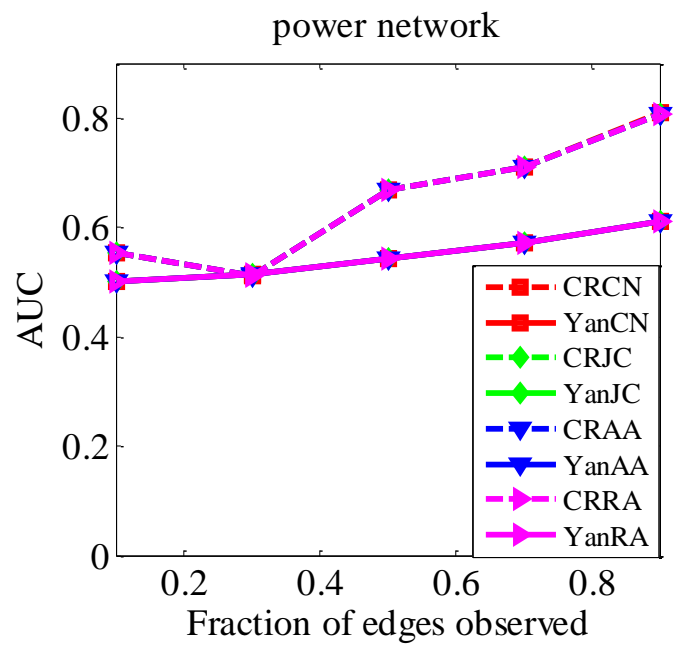

(d)

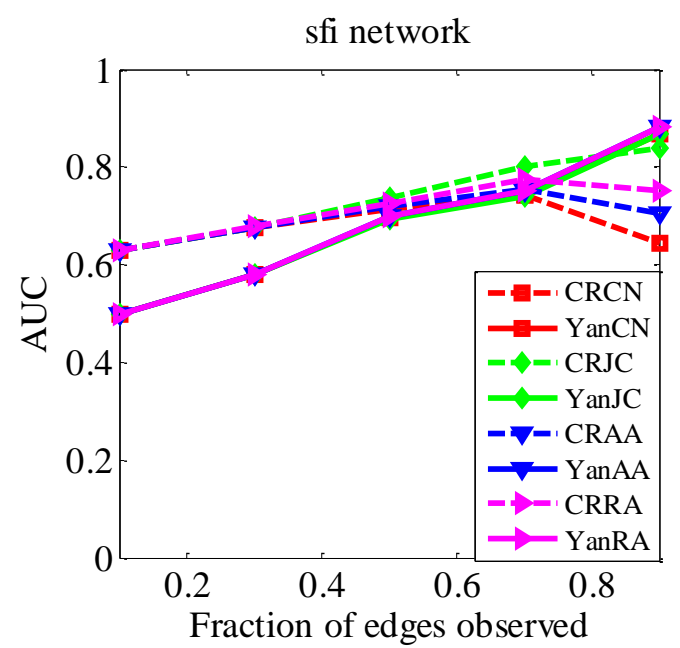

(f)

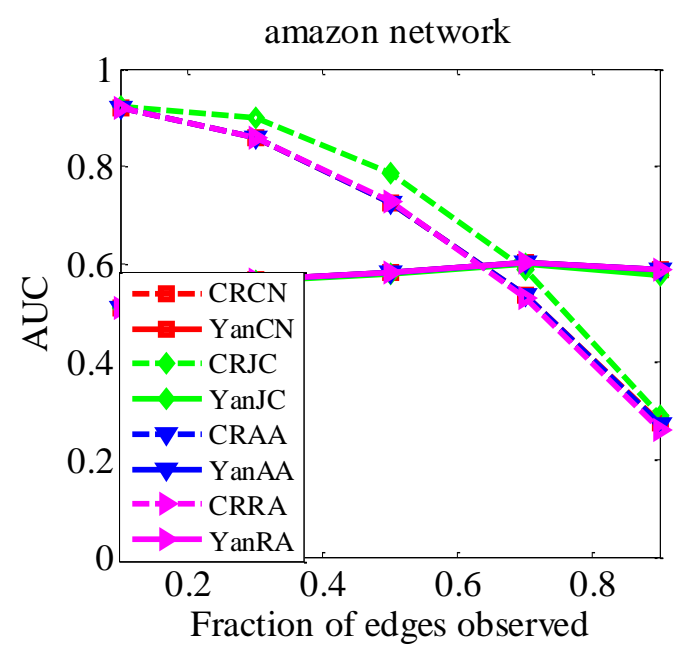

(h) 


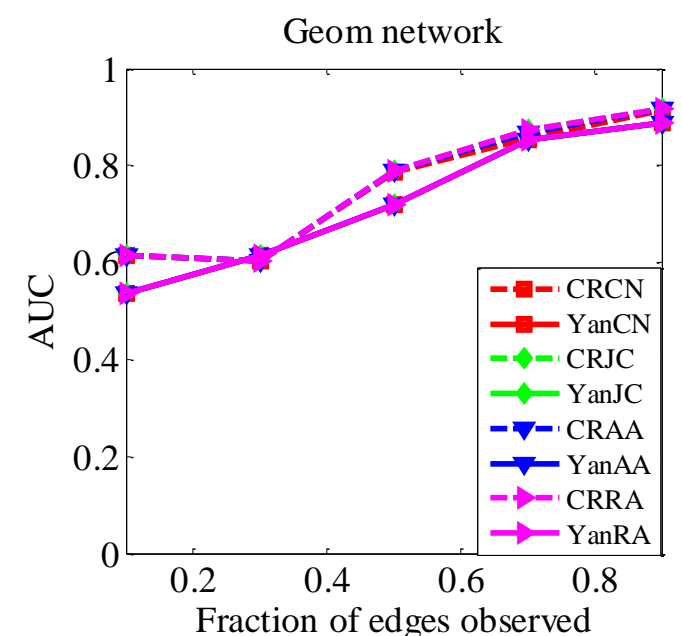

(i)

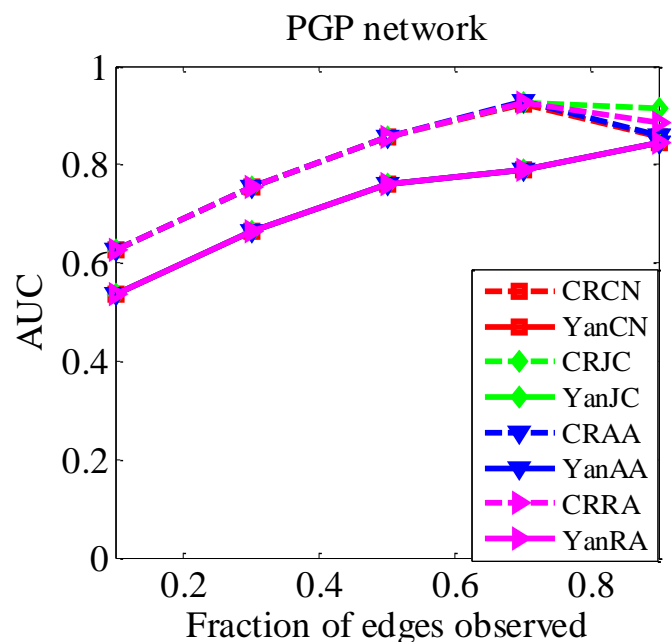

(j)

Fig.9. Plot the prediction results of our methods and Yan's methods. (a), (b) Show the results of benchmark network. Here, (a) $N=100, \mu=0.05,<k>=10$ and $k_{\max }=25$. (b) $N=1000, \mu=0.05,<$ $k>=4$ and $k_{\max }=10 .(\mathrm{c}),(\mathrm{d}),(\mathrm{e}),(\mathrm{f}),(\mathrm{g}),(\mathrm{h}),(\mathrm{i}),(\mathrm{j})$ Show the results of real-world networks. The red dashed line represents the CRCN algorithm; The red solid line indicates the Yan_CN algorithm; The green dashed line represents the CRJC algorithm; The green solid line represents the Yan_JC algorithm; The blue dashed line represents the CRAA algorithm; The blue solid line represents the Yan_AA algorithm; The rose red dashed line represents the CRRA algorithm; The rose red solid line represents the Yan_RA algorithm;

From above figures we get that at the same time complexity, the prediction accuracy of our algorithm is better than Yan's method. It's because we not only consider the community structure of the network but also the relevance of the communities. Now we calculate the mean values of the ratio of the AUC predicted by different algorithms under different proportions of observations. And give them in table 10. The value of degree exceeds 1 indicates how much better the proposed algorithm performs than Yan's algorithm.

\section{Table 10}

The mean values of the ratio of the AUC predicted by different algorithms under different proportions of observations

\begin{tabular}{ccccc}
\hline \hline Network & CRCN/YanCNCRJC/YanJCCRAA/YanAACRRA/YanRA \\
\hline Karate & 0.9002 & $\mathbf{1 . 0 6 3 4}$ & 0.8986 & 0.9175 \\
terrorist & 0.8849 & $\mathbf{1 . 0 0 4 5}$ & 0.9018 & 0.9242 \\
dolphins & 0.9803 & $\mathbf{1 . 0 6 5 7}$ & 0.9830 & $\mathbf{1 . 0 0 8 0}$ \\
lemis & 0.8168 & 0.9992 & 0.8400 & 0.8830
\end{tabular}




\begin{tabular}{|c|c|c|c|c|}
\hline grassland & 1.0440 & 1.1013 & 1.0519 & 1.0678 \\
\hline football & 0.8495 & 1.0011 & 0.8676 & 0.9094 \\
\hline sfi & 1.0362 & 1.1070 & 1.0512 & 1.0690 \\
\hline jazz & 0.8705 & 0.9494 & 0.8708 & 0.8777 \\
\hline USAirline & 0.9497 & 0.9848 & 0.9535 & 0.9629 \\
\hline $\mathrm{ssc}$ & 1.1649 & 1.1684 & 1.1658 & 1.1667 \\
\hline email & 1.0119 & 1.0229 & 1.0099 & 1.0131 \\
\hline polblogs & 0.9663 & 0.9694 & 0.9677 & 0.9717 \\
\hline netscience & 1.0533 & 1.0568 & 1.0544 & 1.0550 \\
\hline protein & 1.2092 & 1.2095 & 1.2100 & 1.2091 \\
\hline amazon & 1.1859 & 1.2477 & 1.1824 & 1.1773 \\
\hline power & 1.1797 & 1.1789 & 1.1789 & 1.1789 \\
\hline Geom & 1.0509 & 1.0557 & 1.0545 & 1.0557 \\
\hline PGP & 1.1232 & 1.1381 & 1.1263 & 1.1309 \\
\hline 100_0.05 & 0.7762 & 0.9995 & 0.8277 & 0.8877 \\
\hline 100_0.10 & 0.7359 & 1.0549 & 0.7543 & 0.7933 \\
\hline 100_0.20 & 0.6945 & 1.0145 & 0.7039 & 0.7573 \\
\hline 100_0.30 & 0.9310 & 0.9998 & 0.9449 & 0.9612 \\
\hline 100_0.40 & 0.9833 & 1.0131 & 0.9848 & 0.9962 \\
\hline $100 \_0.50$ & 1.0074 & 1.0221 & 1.0146 & 1.0303 \\
\hline $1000 \_0.05$ & 1.0811 & 1.0851 & 1.0817 & 1.0840 \\
\hline 1000_0.10 & 1.0576 & 1.0650 & 1.0581 & 1.0625 \\
\hline $1000 \_0.20$ & 1.0575 & 1.0636 & 1.0587 & 1.0620 \\
\hline 1000_0.30 & 1.0640 & 1.0726 & 1.0659 & 1.0696 \\
\hline 1000_0.40 & 1.0711 & 1.0748 & 1.0719 & 1.0719 \\
\hline $1000 \_0.50$ & 1.0367 & 1.0388 & 1.0375 & 1.0376 \\
\hline
\end{tabular}

This chapter showed us the performance of our algorithm mainly from the accuracy, the time complexity and the universality. From above results we can see that the performance is not good enough if the algorithm only consider the similarity of nodes or the community structure in the traditional sense to predict missing links. And the prediction results that obtained by the combination of these two information are worse than our algorithm's which add the community relevance information. Finally, we found our algorithm is effective and stable.

\section{Conclusion}

It must be emphasized that not only a new index, our focus is on the new philosophy in the formulation of community-based indices for network science and potential applications. We advocate a shift in perspective from nodes similarity to community relevance. In fact, the concrete form of community relevance can be varied from different perspectives. The performance of the algorithm proposed in this paper is better than other methods when the networks with high modularity $(\mathrm{Q})$. 
Otherwise the community relevance matrix which obtained from the community structure information is wrong. This will result in the invalidation of the algorithm. Moreover, the performance of our algorithm is better when the degree distributions are consistent under different proportion of observations. It's because that if the degree distributions of the network are not consistent, the structure of the network is not stable enough. The network may have inconsistent information which will influence the community division. Inaccurate community structure information will lead to the poor performance of our prediction method. To summarize, our approach is more suitable for the community networks which the degree distributions are consistent. Finally, our method is more efficient than other node similarity algorithms when the fraction of observed edges is small. Especially, when the observed parts are less than $30 \%$, the accuracy of our algorithm will improve about $10 \%-20 \%$ than traditional similarity methods. The results indicate that under the same time complexity, our algorithm with higher prediction accuracy. That's because the reduction of the training set will directly affect the accuracy of the neighborhood indices seriously, but the impact on the community relevance indices was not so great. Especially, when the data is typical and sparse our method is extremely fast.

The next work we want to deal with the dynamic networks. It requires us to convert the prediction model, and to changes the existing community relevance definition according to the characteristics of dynamic networks.

\section{Acknowledgements}

The work was supported in part by the National key basic research development program 2013CB329402, the National Science Foundation of China Grants 61473215, the discipline innovative engineering plan (111 plan): No. B07048, the major research plan: 91438201 and 91438103, the Ministry of education "the Yangtze River scholars and innovation team development plan": IRT1170.

\section{Reference}

[1] T. Zhou, L. Lu, Link prediction in complex networks: A survey, Phys. A, 390 (2011)1150-1170.

[2] L. Getoor, C. P. Diehl, Link mining: a survey, SIGKDD Explorations, 7 (2005)3-12.

[3] W. Q. Wang, Q. M. Zhang, T. Zhou, Evaluating network models: A likelihood analysis, EPL, 92 (2012)28004.

[4] C. V. Cannistraci, G. Alanis-Lobato, T. Ravasi, From link-prediction in brain connectomes and protein interactomes to the local-community-paradigm in complex networks, Sci. Rep. UK, 3 (2013)1613. 
[5] Q. M. Zhang, L. Lu, W. Q. Wang, Y. X. Zhu, T. Zhou, Potential Theory in Directed Networks, PLOS ONE, 8 (2012)e55437.

[6] F. Xie, Z. Chen, J. X. Shang, X. P. Feng, J. Li, A link prediction approach for item recommendation with complex number, Knowledge-Based Syst. (2015).

[7] D. Lin, An information-theoretic definition of similarity, In Proc. 15thInt. Conf. Mach. Learn, San Francisco: Morgan Kaufman Publishers, (1998)296-304.

[8]D. L. Nowell, J. Kleinberg, The link prediction problem for social networks, In Proc.12thInt.Conf. Inform. Know. Manage, CIKM ’03, NY, USA, ACM, (2003)56-559.

[9] A. Clauset, C. Moore, M. E. J. Newman, Hierarchical structure and the prediction of missing links in networks, Nature, 453, (2008)98-101.

[10] M. A. Hasan, V. Chaoji, S. Salem, M. Zaki, Link prediction using supervised learning, In Proc. of SDM 06 workshop on Link Analysis, Counterterrorism and Security, (2006).

[11] K. Subbian, P. Melville, Supervised rank aggregation for predicting influence in networks, In Proc. of IEEE Conf. on Social Computing, (2011)661-665.

[12] L. Lu, T. Zhou, Link prediction in weighted networks: The role of weak ties, EPL, 89 (2010)18001.

[13] J. Kunegis, J. Fliege, Predicting Directed Links using Non-diagonal Matrix Decompositions, IEEE 12th Inter. Conf. Data Mining, (2012)948-953.

[14] M. S. Pardo, R. Guimerà, L. A. N. Amaral, Extracting the hierarchical organization of complex systems, Proc. Natl. Acad. Sci. USA, 104 (2007)15224-15229.

[15] M. Girvan, M. E. J. Newman, Community structure in social and biological networks, Proc. Natl. Acad. Sci. USA. 99 (2002) 7821-7826.

[16] R. Guimerà, M. S. Pardo, Missing and spurious interactions and the reconstruction of complex networks, Proc. Natl. Acad. Sci. USA. 106 (2009) 22073-22078.

[17] B. Yan, S. Gregory, Finding missing edges in networks based on their community structure, Phys. Rev. E. 85 (2012)056112.

[18] J. Y. Ding, L. C. Jiao, Y. T. Hou, J. S. Wu, Y. T. Qi, Prediction of missing links based on multi-resolution community division, Phys. A, 417 (2014)76-85.

[19] L. Y. Lü, L. M. Pan, T. Zhou, Y. C. Zhang, H. E. Stanley, Toward link predictability of complex networks, Proc. Natl. Acad. Sci. USA. 112 (2015)2325-2330. 
[20] M. E. J. Newman, M. Girvan, Finding and evaluating community structure in networks, Phys. Rev. E. 69 (2004)026113.

[21] Z. Zhao, S. Feng, Q. Wang, J.Z. Huang, G.J. Williams, J. Fan, Topic oriented community detection through social objects and link analysis in social networks, Knowledge-Based Syst. 26 (2012)164-173.

[22] P. D. Meo, E. Ferrara, G. Fiumara, A. Ricciardello, A novel measure of edge centrality in social networks, Knowledge-Based Syst. (2012)136-150.

[23] Z. Y. Xia, Z. Bu, Community detection based on a semantic network, Knowledge-Based Syst. 26 (2012)30-39.

[24] B. Amiri, L. Hossain, J. W. Crawford, R. T. Wigand, Community detection in complex networks: multi-objective enhanced firefly algorithm, Knowledge-Based Syst. 46 (2013)1-11.

[25] J. S. Wu, Y. Jiao, Clustering dynamics of complex discrete-time networks and its application in community detection, Chaos, Vol. 24 (2014)033104.

[26] J. S. Wu, Y. T. Hou, Y. Jao, Y. Li, X. X Li, L. C. Jiao, Density shrinking algorithm for community detection with path based similarity, Phys. A, 433 (2015)218-228.

[27] J. S. Wu, F. Wang, P. Xiang, Automatic network clustering via density-constrained optimization with grouping operator, Applied Soft Computing, 38 (2016)606-616.

[28] R. Guimera, L. A. N. Amaral, Functional cartography of complex metabolic networks, Nature, 433 (2005)895-900.

[29] J. Duch., A. Arenas, Community detection in complex networks using Extremal Optimization, Phys. Rev. E. 72 (2005)027104.

[30] M. E. J. Newman, Finding community structure in networks using the eigenvectors of matrices, Phys. Rev. E. 74 (2006)036104.

[31] S. Fortunato, M. Barthélemy, Resolution limit in community detection, Proc. Natl. Acad. Sci. USA. 104 (2007) 36-41.

[32] M. Rosvall, C. T. Bergstrom, An information-theoretic framework for resolving community structure in complex networks, Proc. Natl. Acad. Sci. USA. 104 (2007)7327-7331.

[33] M. Chen, T. Nguyen, and B. K. Szymanski, On measuring the quality of a network community structure, in Proc. ASE/IEEE Int. Conf. Social Comput., Washington, DC, USA, Sep., (2013)122-127.

[34] S. G. Liu, Detecting communities of directed networks via a local algorithm, J. Math. Inform. 1 (2013)43-51. 
[35] J. W. Chang, C. Chen, CROP: Community-Relevance-Based Opportunistic Routing in Delay Tolerant Networks, IEEE Wireless Communications and Networking Conf. (WCNC): NETWORKS, (2013)1938-1943

[36] X. Feng, Link prediction in complex networks: a clustering perspective, Eur. Phys. J. B, 85 (2012)3.

[37] S. Zhou, R. J. Mondrag, The rich-club phenomenon in the Internet topology, IEEE Commun. Lett. $8(2004) 180$

[38] M. E. J. Newman, Clustering and Preferential attachment in growing networks, Phys. Rev. E. 64 (2001) 025102.

[39] G. Salton, M. J. McGill, Introduction to Modern Information Retrieval, McGraw-Hill, (1983).

[40] L. A. Adamic, E. Adar, Friends and neighbors on the web, Social Net. 25 (2003)211-230.

[41] T. Zhou, L. Lu, Y. C. Zhang, Predicting missing links via local information. Eur. Phys. J. B 71 (2009)623-630

[42] J. A. Hanley, B. J. McNeil, The meaning and use of the area under a receiver operating characteristic (ROC) curve, Radiology, 143 (1982)29-36.

[43] T. Fawcett, An introduction to roc analysis, Patter Recognition Letts. 27 (2006)861-874.

[44] A. Lancichinetti, S. Fortunato, F. Radicchi, Benchmark graphs for testing community detection algorithms, Phys. Rev. E. 78 (2008)046110.

[45] A. Lancichinetti, S. Fortunato, Community detection algorithms: a comparative analysis, Phys. Rev. E. 80 (2009)056117.

[46] L. J. Ma, M. G. Gong, J. Liu, Q. Cai, L. C. Jiao, multi-level learning based memetic algorithm for community detection, Appl. Soft. Comput. 19 (2014)121-133.

[47]N. Blagus, S. Lovro, B. Marko, Self-similar scaling of density in complex real-world networks, Phys. A, 391 (2012)2794-2802.

[48] A. Clauset, M. E. J. Newman, C. Moore, Finding community structure in very large networks, Phys. Rev. E. 70(2004)066111. 